\title{
Les travaux d'aménagement de la région du Bas-Rhône et du Languedoc
}

\section{Development work in the Bas-Rhône and Languedoc Region}

\author{
par M. BAUZIL \\ INGÉNIEUR EN CHEF DES PONTS ET CHAUSSÉES, \\ DIRECTEUR DES ÉTUDES ET TRAVAUX \\ DE LA COMPAGNIE NATIONALE D'AMÉNAGEMENT DU BAS-RHONE ET DU LANGUEdOC
}

La Compagnie Nationale d'Aménagement de la Région du Bas-Rhône et du Languedoc a été créée par décret du 3 février 1955.

Elle est chargée de la mise en valeur, par l'irrigation, des plaines qui, entre le littoral méditerranéen et le pied des garrigues calcaires, s'étend de la rive droile du Rhône à la région du Minervois, i louest de Narbonne.

Cet ensemble sera desservi par dema systemes d'irrigation distincts.

Le premier (périmètre Est) tirera son aliment du Rhcine sur lequel la Compagnie concessionnaire est autorisée à prélever un debit maximum de $75 \mathrm{~m}^{3} / \mathrm{s}$. II dominera une surface de $129.100 \mathrm{ha}$.

Le second (périmètre Onest) utilisera les eanx du feuve orb, grossies de celles qu'un des prochains equipements hydrólectriques d'E.D.F. doit permettre de dériver du versant atlantique dans le bassin méditerranéen.

Ce deuxieme périmetre couvrira d'abord ane section de 30.000 ha en rive droite de l'Orb, mais pourra s'étendre par la suite à près de 80.000 ha, moyennant la constitution de réserves complémentaires sur l'Orb et l'utilisation conjointe d'une partie des débits ac l'Aude.

L'irrigation du périmètre Est nécessite le relèvement des eaux, par pompage, d'une part sur le cours même du canal principal, d'autre part pour lalimentation, a partir de ce canal, des branches maitresses qui en dérivent. La premiere et la plus importante de ces stations, située $\dot{a} 12 \mathrm{~km}$ de la prise d'ean au Rhône, absorbera, at stade final de son équipement, une puissance de $39.000 \mathrm{ch}$.

L'irrigation du périmètre Ouest pourra se faire par gravité, mais elle impose, en revanche, la régularisation préalable des débits de l'Orb par des barrages d'accumulation dont la construction sera entreprise au fur et d mesure du développement des irrigations.

D'une façon générale, et, en tout cas, sur les $30.000 \mathrm{ha}$ du secteur des Costières qui, dans le système Est, sera le premier desservi, l'irrigation se fera par aspersion et à la demande, la distribution étant assurée par un réseau de conduites en charge.

Les travaux d'aménagement du périmètre Est sont en cours d'exécution.

Ceux du périmètre ouest commenceront très prochainement
The Compagnie Nationale d'Amenagement de la Région du Bas-Rhóne et du Languedoc (State Company for the Development of the BasRhone and Languedoc Region) was set up by the Govermment on February $3 \times 1005$.

This company's aim is to atse irrigation methods to develop the plains which run from the right bank of the Rhone to the Minervois region near Narbonne and which lie between the Mediterranean coast and the foot of the limestone garrigues.

This region will be covered by two irrigation systems.

The first (the eastern sector) will be fed by the Rhone from which the company is allowed to dratv a maximum of 75 cubic metres per second. It will cover an area of 129,000 hectares.

The second (the western sector) will use water from the River Orb the discharge of which will be increased by water from an Electricité de France hydroelectric project in which water is diverted from the Atlantic watershed to the Mediterranean.

At first, the second sector will cover 30,000 hectares on the right bank of the Orb, but it will be possible to increase it later to 80,000 provided that reserves are built up on the Orb and provided that part of the flow of the river Aude is also used.

Pumps will have to be used to irrigate the eastern sector. Water will have to be pumped into the main canal and then into the main secondary canals which run from it. The first. and largest pumping station, which is located 12 kilometres from the offtake on the Rhone will absorb $39,000 \mathrm{HP}$ in its final state.

The western sector can be irrigated by gravity, but the flow of the Orb will first have to be controlled by storage dams to be built as the irrigated area is extended.

Generally speaking, intermittently operating sprinkler irrigation will be used. Supply will be from a network of pressure pipelines. This method will defnitely be used for the 30,000 hectare Costieres region, which will be the first section to be irrigated.

Work is in progress in the eastern sector.

Work will start on the unestern sector in the near future. 


\section{LA COMPAGNIE NATIONALE D'AMENAGEMENT DU BAS-RHONE ET DU LANGUEDOC}

La Compagnie Nationale d'Aménagement de la Région du Bas-Rhòne et du Languedoc a été crééc par décret du 3 février 1955.

Elle s'est substituée à une société d'études constituée à Nîmes en janvier 1952 dans le but de mettre sur pied un avant-projet d'irrigation des plaines du Bas-Rhône-Languedoc et d'analyser toutes les conséquences financières, économiques et sociales qu'entraînerait l'exécution d'un tel projet, non seulemrent dans les zones directement intéressées par l'irrigation, mais aussi dans celles limitrophes de garrigues et de montagnes, dont l'économie, d'ailleurs plus ou moins liée à celle de la plaine, justifiait une action évolutive parallèle. Organisée en forme de société d'économie mixte, la Compagnie a vu ses statuts approuvés par décret du 27 juillet 1956 . La concession générale des travaux déclarés d'utilité publique pour l'établissement et l'exploitation d'un réseau d'irrigation entre le Rhône et la région de Lézignan-Corbières dans l'Aude lui a été attribuée par décret du 14 septembre 1956 .

Les crédits d'investissement nécessaires à la réalisation des travaux ont été prévus par le décret-programme du 20 mai 1955 pour l'exécution du deuxième plan de modernisation et d'équipement de l'Agriculture.

Les dépenses d'investissement sont subventionnées au taux de $60 \%$ de leur montant, les subventions étant intégralement versées en capital.

Les sommes non couvertes par les subventions font l'objet de prêts à caractéristiques spéciales du fonds de développement économique et social, prêts consentis à des conditions particulièrement favorables puisqu'amortissables en 35 ans au taux global et forfaitaire de $4 \%$, intérêts et amortissements compris, les cinq premières années ne comportant que le versement d'un intérêt de $1,25 \%$. Ces avantages financiers sont consentis à raison de l'intérêt qui s'attache à libérer le Midi viticole de l'impasse économique où il se trouve présentement engagé, et du fait bien connu que la bonification agraire procurée par l'irrigation, si elle est toujours payante, ne l'est généralement qu'à terme, les transformations de structure et de méthodes qu'elle postule ne pouvant être que l'œuvre d'une génération.

La Compagnie a son siège à Nî̀mes.

Elle comprend :

- Une Direction générale,

- Une Division des affaires administratives et financières,

- Une Direction des Travaux généraux, chargée de l'étude et de la réalisation de l'ossature du système d'irrigation : prise au Rhone, canaux principaux et branches maîtresses dérivées, barrages d'accumulation et de dérivation, stations de pompage principales, gros travaux d'infrastructure divers,

-. Une Direction de l'Hydraulique agricole et de la mise en valeur, dont dépendent les études et enquêtes agropédologiques liminaires, l'élaboration des projets et la réalisation des réseaux de distribution, l'exploitation des réseaux et les tâches complémentaires de l'irrigation : expérimentation, vulgarisation agricole spécialisée, etc. A cette Direction, sont rattachés deux services dont l'activité intéresse les zones de garrigue et de montagne au moins autant que les secteurs à irriguer : un service forestier et un service de l'habitat rural et des aménagements communaux.

Le premier est chargé de l'étude du reboisement en zone de garrigue et de montagne dans les terrains privés ou communaux et du développement, sur les morts terrains du périmètre irrigable, de plantations arbustives à évolution rapide orientées vers la production de bois de papeterie et de caisserie.

Le second est destiné à apporter son concour's à l'élaboration et à la réalisation d'un programme de rénovation de l'habitat rural dans les secteurs irrigués et les zones périphériques. Son but est, notamment, d'aider les villageois à mettre sur pied des plans de modernisation de leur habitat ou des projets de constructions entièrement nouvelles, de faciliter l'accès aux sources de financement en aidant à la constitution des dossiers nécessaires et de grouper les réalisations en vue d'abaisser les prix de revient et de rendre possible le recours à des techniques modernes de construction. 


\section{LA REGION DU BAS-LANGUEDOC}

Avant de parler des travaux hydrauliques dont la réalisation est envisagée, il y a lieu de les situer rapidement dans leur cadre naturel.

La région du Bas-Rhône-Languedoc a été jusqu'à maintenant vouée à la monoculture de la vigne. La raison en est principalement le climat relativement très sec, l'insuffisance et surtout l'irrégularité des précipitations atmosphériques qui, s'ils ne sont pas compensés par l'irrigation, font peser sur toute autre spéculation agricole de redoutables aléas et la condamnent en tout cas à des rendements insuffisants.

Cette activité viticole a été jusqu'à ces dernières années souvent prospère, nonobstant quelques crises passagères, mais la viticulture languedocienne connaît maintenant - on ne saurait dire qu'elle traverse - une crise grave dont la nature et les origines $\rightarrow$ semblent ruiner tout espoir d'un retour à la prospérité.

Les éléments principaux en sont la concurrence $d u$ vignoble nord-africain et l'intervention sur le marché des néo-viticulteurs du Centre et de l'Ouest. Cette surproduction est désormaisc mondiale ainsi qu'il résulte des travaux de l'Office International du Vín et, de ce fait, la production mondiale, surtout celle des vins de qualité courante, ne peut attendre d'un développement possible des exportations qu'un secours limité, hor's de proportion avec les excédents. Il va de soi, d'autre part, qu'on ne saurait exiger de l'Etat qu'il perpétue le soutien qu'il donne a la viticulture par le biais de la distillation d'une partie des excédents, soutien qui coûte fort cher au budget sans satisfaire pour autant les intéressés et qui ne se serait trouvé justifié que si l'espoir avait pu être gardé d'un retour prochain à l'équilibre entre la production et la consommation.

La région géographique dont la Compagnie du Bas-Rhône-Languedoc envisage l'irrigation s'étend du Rhône à Lézignan, entre le pied des garrigues calcaires qui prolongent vers le sud les derniers contreforts des Cévennes et la mer. Elle s'étend aux départements du Gard, de l'Hérault et de l'Aude. La surface totale dominée par les canaux d'irrigation projetés est d'environ 250.000 ha, dont 130.000 par le canal principal issu du Rhône et 100.000 par les canaux dérivés de l'Orb.

\section{Les sols.}

Les sols du périmètre irrigable sont constitués par des formations alluvionnaires qui évoluent en sols bruns avec ou sans accumulation de calcaire en un point du profil, en sols bruns légèrement lessivés et décalcifiés, en sols hydromorphes dans les dépressions, lorsqu'il y a présence d'une nappe phréatique proche de la surface. Ces sols possèdent, d'une façon générale, une texture moyenne et une fertilité suffisante. Toutefois, ceux qui portent des vignobles manquent le plus souvent d'humus et il est certain que leur reconversion devra s'accompagner d'une amélioration de la structure qui pourra précisément résulter de la diversification des spéculations agricoles, parmi lesquelles des cultures de légumineuses fourragères et d'engrais vert devront trouver place.

Certaines formations géologiques comme les marnes plaisanciennes et les sables de Montpellier donnent naissance à des sols bruns calcaires, à des sols rouges type méditerranéen et à des sols lithochromes qui couvrent une gamme très étendue de productions végétales possibles. Un cas bien particulier concerne les sols issus des cailloutis pliocènes dont la destinée pour l'arboriculture fruitière est remarquable.

Les sols compris dans le périmètre d'irrigation font l'objet d'études systématiques. La Compagnie a monté à Nîmes un laboratoire qui procède à leur analyse physique et chimique et à la détermination des constantes hydrodynamiques : perméabilité, capacité de rétention, dose d'irrigation. Il est prévu qu'un carnet, définissant les qualités du sol, les précautions à prendre dans la conduite de l'irrigation, les doses à ne pas dépasser, le mode d'arrosage le plus convenable et les cultures les mieux appropriées, sera mis à la disposition de chaque exploitant.

\section{Le climat.}

Le facteur principal limitant la production agricole dans le Bas-Languedoc est l'insuffisance des précipitations atmosphériques pendant les mois chauds, d'où résulte la nécessité d'irriguer pour pouvoir entreprendre, avec une garantie suffisante de réussite et de constance dans les rendements, d'autres spéculations agricoles que celle de la vigne.

L'état hygrométrique faible entraîne une évaporation intense et une sécheresse peu favorable à l'agriculture.

La moyenne des températures est supérieure de $3^{\circ}$ à la moyenne générale de la France.

Des minima élevés, joints à une grande luminosité, rendent possible, en culture irriguée, l'obtention de plusieurs récoltes par an. Les températures maxima sont en moyenne de $25^{\circ}$ au printemps, $28^{\circ}$ en été, $15^{\circ}$ en automne et $12^{\circ}$ en hiver, cependant que les minima moyens sont respectivement de $11^{\circ}, 16^{\circ}, 6^{\circ}$ et $3^{\circ}$.

La pluviosité est irrégulière. Les pluies tom- 
bent par grosses averses. Les précipitations annuelles sont de l'ordre de $700 \mathrm{~mm}$ dont, en moyenne, $160 \mathrm{~mm}$ (23 j.) au printemps, $145 \mathrm{~mm}$ (15 j.) en été, $240 \mathrm{~mm}$ (27 j.) en automne et $150 \mathrm{~mm}$ ( $24 \mathrm{j}$ en hiver).

La région est soumise à un régime de vents caractérisé par la fréquence du mistral, vent froid et desséchant qui souffle du nord-est et du nord, nord-ouest surtout en hiver et en été. Il en résulte deux saisons sèches correspondantes. Dans les périodes de basses pressions atmosphéricues, le vent marin chaud et chargé d'humidité souffle de la Méditerranée, surtout à la fin de l'été. Sa vapeur d'eau, en se condensant sur la barrière cévenole, provoque des orages caractéristiques et de courte durée. Le vent d'ouest, océanique, humide et frais est assez rare.

Le climat de la région se caractérise donc par l'existence de températures élevées pouvant atteindre, en été, $38^{\circ}$ à l'ombre, une luminosité intense, des précipitations irrégulières à forme orageuse et une sécheresșe estivale très marquée. On voit par là que l'irrigation est nécessaire pour compenser l'insuffisance et l'irrégularité des précipitations et qu'elle donnera son plein effet à raison des conditions très favorables de température, et de luminoșité rencontrées dans la région.

\section{Démographie.}

La population comprise dans les limites du périmètre irrigable atteint 579.000 personnes réparties dans 227 communes.

L'exode rural sévit dans la région au profit des villes et en particulier de Nìmes et de Montpellier. C'est ainsi que, de 1936 à 1954, la popula- tion de Montpellier a augmenté de $8 \%$, alors que celle du département de l'Hérault a diminué de $6 \%$.

Les relevés cadastraux montrent que les propriétés moyennes sont rares, une importante fraction dụ domaine foncier étant extrêmement morcelée.

Une enquête récente a permis de constater que : dans le Gard, $50 \%$ des exploitations sont inférieures à 3 ha et couvrent $9 \%$ du vignoble, alor's que les exploitations de plus de 15 ha, qui représentent $6 \%$ du nombre total, couvrent $58 \%$ de la superficie. Il en est de même dans l'Hérault où $70 \%$ des exploitations couvrent $20 \%$ du vignoble alor's que les exploitations de plus de 15 hectares, soit $3 \%$ du total, couvrent près de $50 \%$ de la superficie.

\section{Agriculture.}

Nous avons déjà dit que le développement de la vigne dans le Bas-Languedoc était essentiellement la conséquence de la séchesse estivale profonde et prolongée. Seule culture actuellement possible, faute d'irrigation sur les coteaux, la vigne a progressivement supplanté, dans les plaines au sol plus frais, toute autre culture. C'est ainsi que, de 1882 à 1956 , la surface plantée en vigne est passée de 18.300 à 88.300 ha dans le Gard et de 69.000 à 178.000 ha dans l'Hérault. Durant cette même période, l'aire réservée aux autres spéculations agricoles n'a cessé de décroître, passant, pour l'ensemble des deux départements précités, de 185.000 à 51.000 ha pour les céréales, de 81.000 à 46.000 ha pour les prairies artificielles et de 54.000 à 18.000 ha pour les prairies naturelles.

\section{LES EQUIPEMENTS HYDRAULIQUES LE SYSTËME D'IRRIGATION DE LA ZONE EST}

\section{Le canal principal.}

Le système d'irrigation de la zone Est est alimenté par le Rhône.

La prise d'eau se situe au mas de Maillan, un peu à l'amont de Fourques (point kilométrique $277+300$ du Rhône), dans une courbe convexe vers la rive droite.

La Compagnie a été autorisée à dériver du fleuve un débit maximum de $75 \mathrm{~m}^{3} / \mathrm{s}$. Ce débit sera modulé en fonction des besoins agricoles.

Pour une campagne agricole fictive composée des mois durant lesquels les conditions de pluviosité minimum ont été observées, les besoins maxima à satisfaire exigeraient que soient dérivés du Rhône les débits suivants :

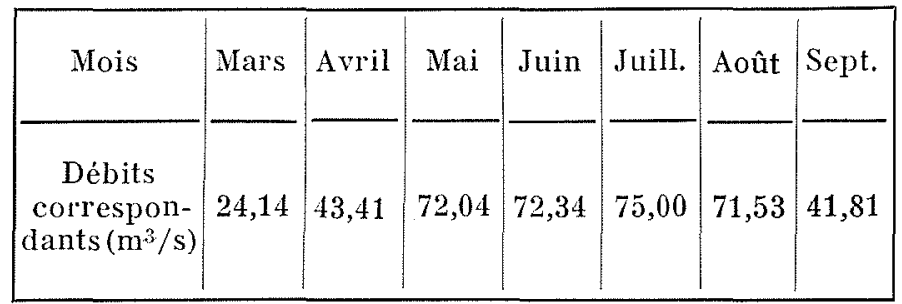

A noter que les chiffres du tableau ci-dessus correspondent à des maxima instantanés. Les débits moyens à prélever mensuellement ne se- 




Fig. 1

L'aménagement hydraulique de la région du Bas-Rhône et du Languedoc.

ront que les $3 / 4$ de ces maxima, étant donné que les irrigations prévues n'auront lieu, en principe, que 18 heures sur 24.

Compte tenu des débits relativement très modestes à dériver durant les mois d'octobre à février pour le maraîchage et surtout pour l'alimentation en eau de quelques centres urbains, le cube total demandé au Rhône sera annuellement en moyenne de l'ordre de grandeur de $550 \mathrm{mil}-$ lions de $\mathrm{m}^{3}$ et au maximum de 750 millions de $\mathrm{m}^{3}$.

Cette évolution des besoins est fort heureusement en harmonie avec les possibilités du Rhône, l'étiage de celui-ci ne se manifestant qu'en septembre-octobre, à une époque où les débits d'irrigation seront en fort déclin ou nuls. Le débit du Rhône à hauteur d'Arles ne descend d'ailleurs que très rarement au-dessous de $600 \mathrm{~m}^{3} / \mathrm{s}$, celui du Petit-Rhône étant environ le $1 / 11^{\circ}$ de celui du cours principal.

L'incidence des prélèvements sur l'évolution de la langue salée sera nulle dans le GrandRhône et certainement très faible dans le Petit. Des observations sont d'ailleurs en cours sur ce dernier bras, qui permettront de connaître avec précision l'évolution de la langue salée aux divers états du fleuve et, par conséquent, de prévoir les conséquences des ponctions qui pourront, dans le futur, être faites en amont du point de débranchement du Petit-Rhône (P.K.279+300), non seulement par la Compagnie, mais aussi par d'autres attributaires.

Le débit solide du Rhône pose un problème assez sérieux. O'n n'a encore réuni que peu de données quantitatives à son sujet, mais on sait qu'il est important, surtout quand la Durance est en crue. Des mesures à la turbidisonde sont systématiquement faites depuis un an. Les premiers résultats permettent d'estimer grossièrement que la teneur en sédiments est, en movenne, de $0,1 \mathrm{~g} / 1$ pendant la campagne d'irrigation et qu'elle est susceptible d'atteindre exceptionnellement en crue $11 \mathrm{~g} / 1$, la décrue solide étant néanmoins très rapide.

Etant donné la vitesse relativement faible de l'eau dans le premier bief du canal principal, $0,75 \mathrm{~m} / \mathrm{s}$ à plein régime, - vitesse qui tombe d'ailleurs à $0,40 \mathrm{~m} / \mathrm{s}$ au-delà du premier kilomètre et qui, étant donné le système à commande par l'aval adopté pour le réglage de la ligne d'eau, sera encore bien moindre au début et en fin de campagne d'irrigation, - il ne faut pas compter qu'une proportion importante des sédiments en suspension dans l'eau dérivée pourra être transportẻe jusque sur les champs. En fait, 
la plus grosse part se déposera dès l'origine. Il y aura d'ailleurs lieu de favoriser ce dépôt dans le chenal de prise précédant l'ouvrage de garde et de régulation, où la vitesse d'approche n'est que de $0,20 \mathrm{~m} / \mathrm{s}$ et d'où ils pourront être facilement repris à la drague et remis en circulation dans le fleuve.

La prise étant faite directement au Rhône sans barrage de dérivation, on ne disposera, en tout cas, d'aucune charge pour chasser hydrauliquement les sédiments décantés et force sera d'entretenir par dragage l'avant-canal et éventuellement le canal lui-même, sur les quelques centaines de mètres où les atterrissements les plus importants sont susceptibles de se produire.

L'ouvrage de prise est incorporé dans la digue marginale de la rive droite qui, en ce point, est distante de $300 \mathrm{~m}$ environ de l'épi longitudinal bordant le lit mineur. Le seuil de prise, établi dans l'alignement de cet épi, est arasé à la cote - 2,00 (la cote d'étiage du Rhône étant $+0,95$ et la cote des plus hautes eaux $+9,00)$. Le lit majeur est traversé par un avant-canal convergent, dont la largeur au plafond va décroissant depuis le seuil de prise, où elle est de $185 \mathrm{~m}$, jusqu'à l'ouvrage de garde et de régulation, où elle n'est plus que de $21 \mathrm{~m}$. Cet avant-canal est bordé de deux digues insubmersibles à leur enracinement sur la digue marginale, mais plongeant légèrement vers le Rhône et donc susceptibles d'être submergées par les crues les plus impor- tantes. Elles sont prołégées, en conséquence, par des perrés maçonnés et des tapis d'enrochements et se terminent côté fleuve par deux musoirs puissamment défendus.

L'ouvrage de garde et de régulation comporte deux pertuis de 2,25 $\mathrm{m}$ de hauteur sur 6,70 $\mathrm{m}$ de largeur, obturés par des vannes-segment automatiques maintenant à leur aval un niveau quasi constant $(+1,10$ à plein débit et $+1,30$ à débit nul). Les pertuis peuvent également être obturés par des batardeaux susceptibles d'être mis en place devant les grilles par l'engin dégrilleur lui-même.

De son origine jusqu'à Pichegu (P.K. 12+547) le canal traverse les plaines relativement très basses du couloir de Saint-Gilles, compris entre le Petit-Rhòne et le pied des Costières du Gard. La faiblesse de la pente motrice disponible et la médiocre qualité des terrains dans lesquels il est creusé, ont conduit à lui donner un gabarit considérable : largeur au plafond : $25 \mathrm{~m}$, talus à 3 de base pour 1 de hauteur, coupés d'une risberme de $2 \mathrm{~m}$ à $1,30 \mathrm{~m}$ sous la ligne d'eau à débit maximum, pistes bordières de $8 \mathrm{~m}$ en rive droite et $10 \mathrm{~m}$ en rive gauche. Au total, compte tenu des cavaliers et des contre-canaux nord et sud nécessaires pour assurer l'écoulement des eaux de drainage de la plaine basse que le canal coupe en deux, la largeur d'emprise sera de $200 \mathrm{~m}$, la largeur au plan d'eau, pour une profondeur normale de $4 \mathrm{~m}$, étant de $53 \mathrm{~m}$.

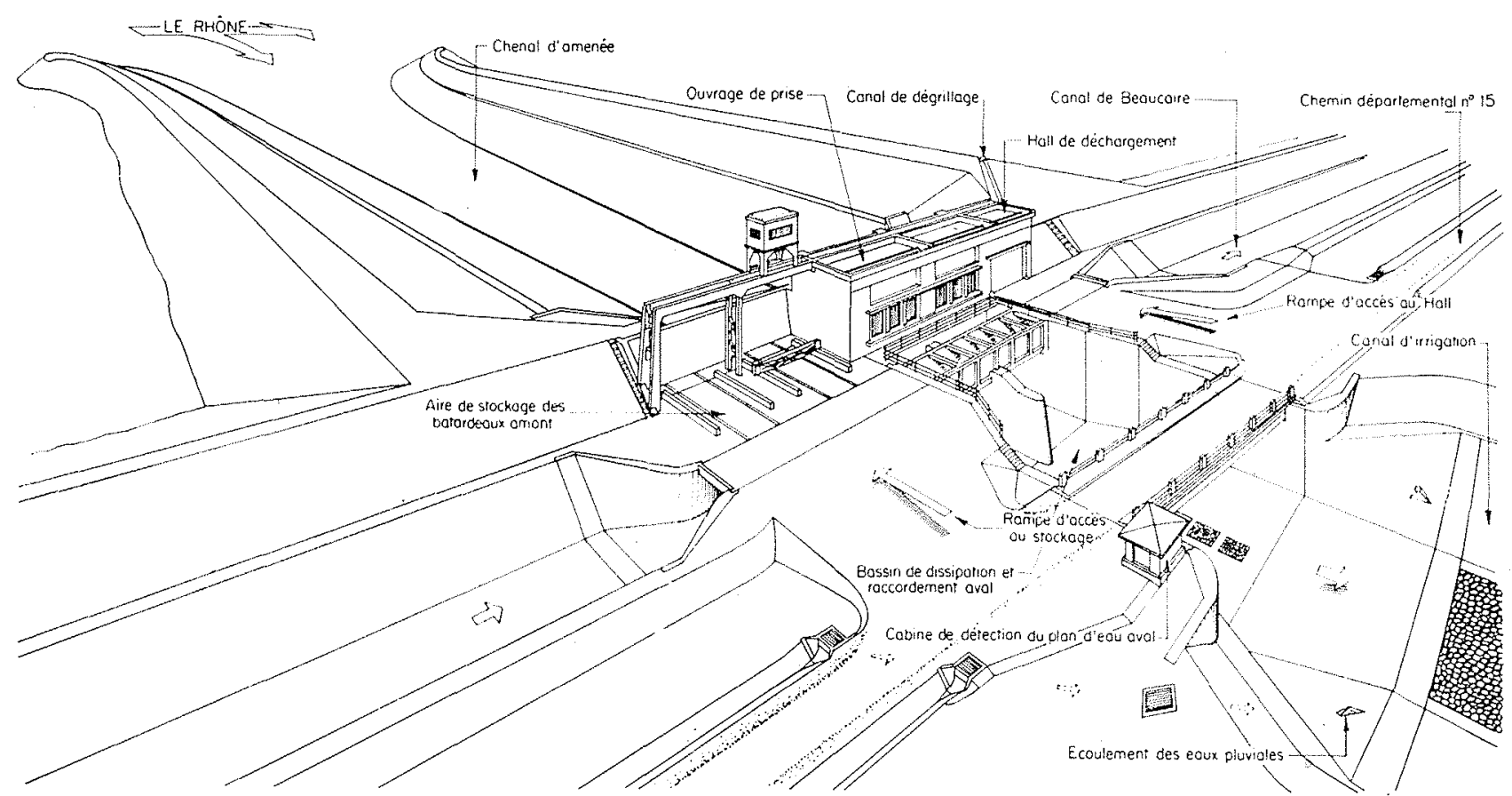

FIG. 2

Ouvrage de régulation à la digue du Rhône. Perspective d'ensemble, vue rle l'aval. 


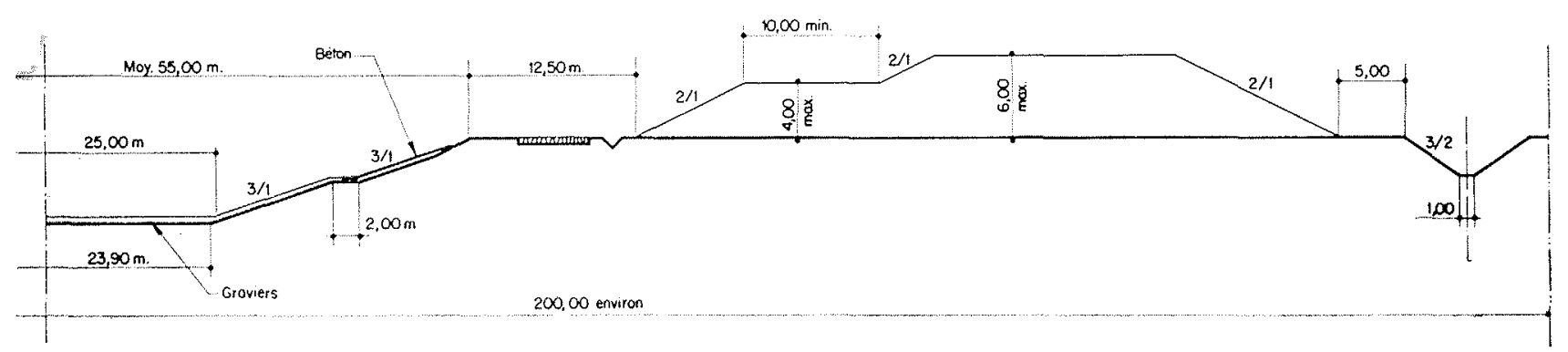

Fiti, 4

Canal principal, de Fourques à Pichegu. Demi-profil en travers. Rive droite.

Sur cette partie de son cours le canal ne recevra qu'un revêtement de batillage régnant entre la cote $+1,80$ (supérieure à $0,50 \mathrm{~m}$ à celle de la ligne d'eau horizontale à débit nul) et la risberme de $2 \mathrm{~m}$ coupant les talus à $1,30 \mathrm{~m}$ audessous de la ligne d'eau à débit maximum, cette risberme étant elle-même protégée par un retour du revètement sur $1,25 \mathrm{~m}$ de largeur. Ce revêtement sera en béton de ciment coulé en place. Il aura $0,12 \mathrm{~m}$ d'épaisseur et sera établi sur une couche de matériaux drainants de $0,30 \mathrm{~m}$ d'épais- seur. La partie inférieure des talus et le radier seront protégés par un lit de graviers d'épaisseur minimum égale à $0,30 \mathrm{~m}$ en talus et $0,50 \mathrm{~m}$ en radier. Compte tenu de ces dispositions, le coefficient de rugosité moyen ( $n$ de la formule de Manning) a été pris égal à 0,025 .

Le terrain encaissant est essentiellement argileux et silteux. Ses caractéristiques mécaniques sont médiocres (angle de frottement interne de 2 à $3^{\circ}$ - cohésion de l'ordre de $2 \mathrm{t} / \mathrm{m}^{2}$ ), mais il est relativement très imperméable et il est

Fig. 5. - Canal principal, tronçon le Rhône-Pichegu. Vue des draglines utilisées pour l'exécution des terrassements.

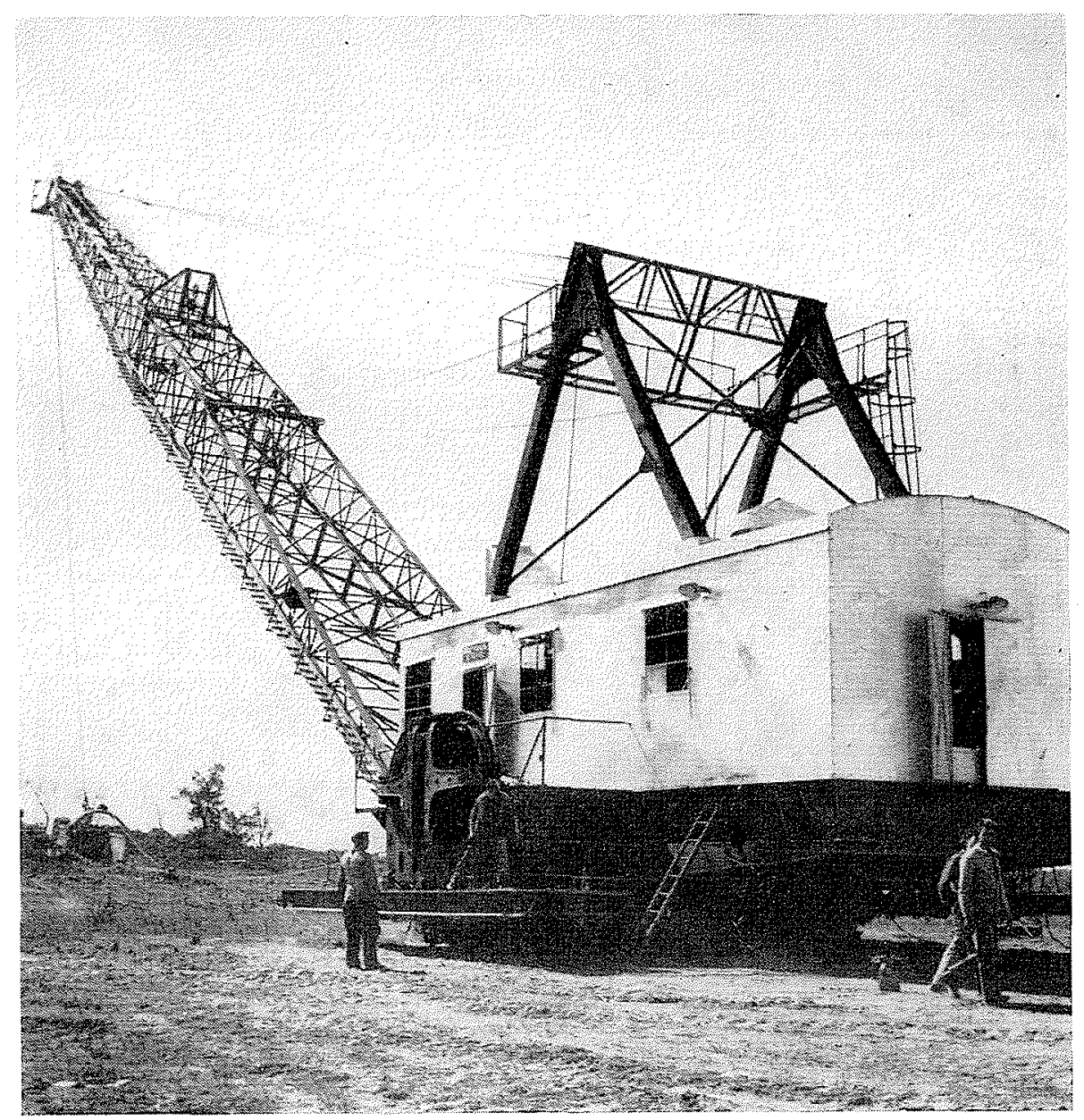




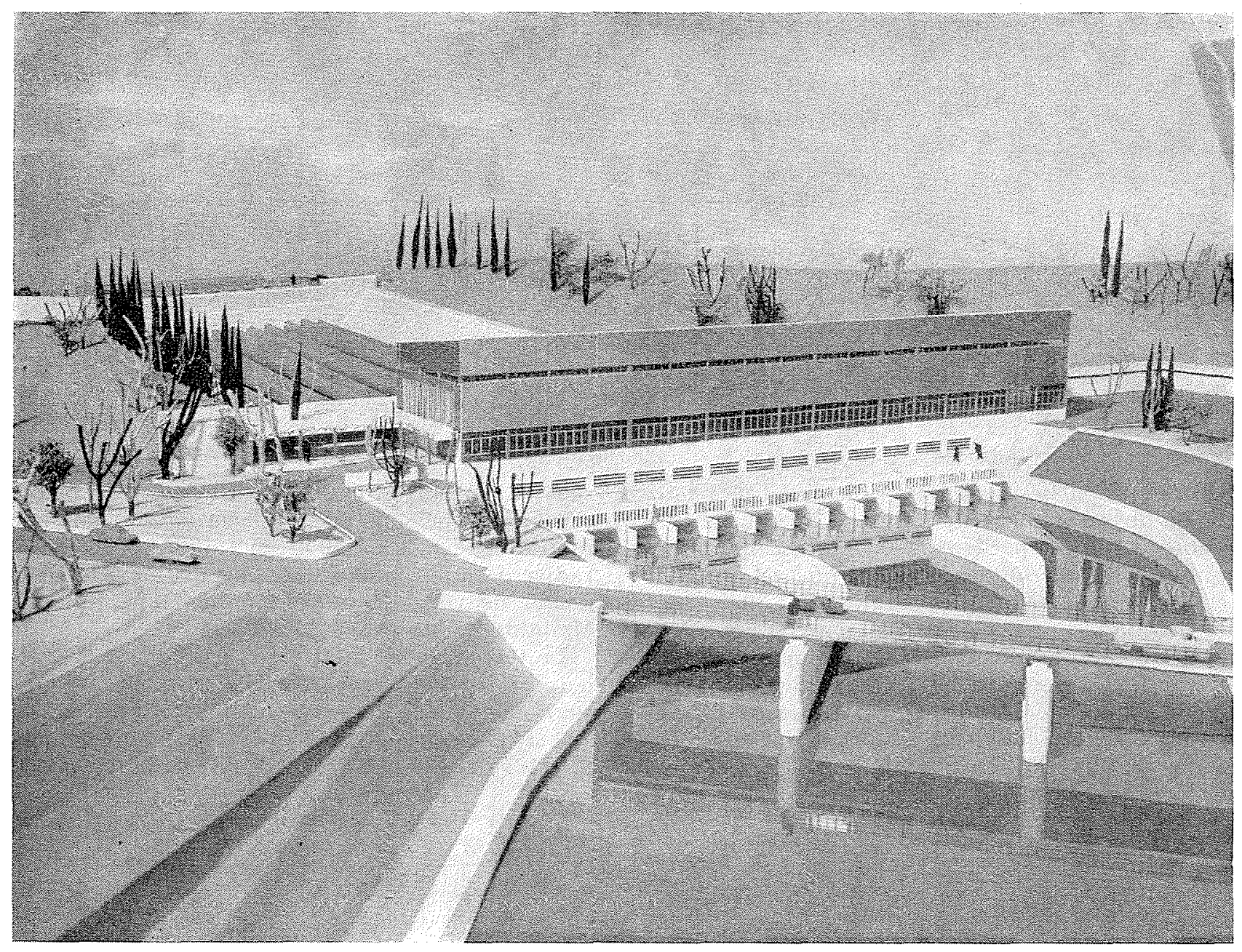

Fra. 6

Station de pompage de Pichegu (maquette).

certain que, malgré l'absence d'un revêtement généralisé, les pertes resteront très faibles et pratiquement sans influence, compte tenu de l'existence des contre-canaux, sur la nappe phréatique des terrains avoisinants.

Sans constituer à proprement parler une tête morte, le canal, sur les douze premiers kilomètres de son parcours, ne desservira, entre son origine et Pichegu, que l'étroit couloir compris entre le Petit-Rhône et le canal de navigation de Beaucaire à Sète. Ce n'est qu'à partir de Pichegu que, soit directement, soit par l'intermédiaire des branches maîtresses qui en dériveront, il assurera l'irrigation de vastes périmètres.

A Pichegu sera installée une importante station de pompage, dans le double but de relever la ligne d'eau du canal principal de la cote $+0,44$ (cote du plan d'eaù à débit maximum) à la cote $+20,33$ et de refouler à la cote $+66,95$ le débit nécessaire à l'irrigation des 34.000 ha constituant les périmètres des Costières et de la Vistrenque. Cette station sera équipée, au stade final :
- Pour le relevement des eaux du camal principal : de 9 pompes de $7.000 \mathrm{l} / \mathrm{s}$ chacune et d'une pompe de $1.600 \mathrm{~J} / \mathrm{s}$ destinée à maintenir en service le canal d'octobre à mars, en dehors de la période des irrigations, cette obligation résultant de la nécessité de ne pas interrompre l'alimentation d'un certain nombre de centres urbains, Montpellier et Sète notamment.

- Pour l'irrigation des plateaux des Costières et de la Vistrenque : de 6 pompes de $2.2501 / \mathrm{s}$ chacune.

Le génie civil de la station, actuellement mis au concours, sera dès l'abord réalisé en vue de sa destination future, mais il n'a été commandé en première étape que : 4 des pompes de 7.000 $\mathrm{l} / \mathrm{s}$, la pompe de $1.600 \mathrm{l} / \mathrm{s}$ et 3 des pompes de $2.250 \mathrm{l} / \mathrm{s}$.

La puissance installée au stade final sera de $38.900 \mathrm{ch}$. Le poste de transformation situé à proximité immédiate de la station sera équipé de trois transformateurs de $20 \mathrm{MVA} 63.000 \times 5.500 \mathrm{~V}$ (cette dernière tension étant celle d'alimentation 


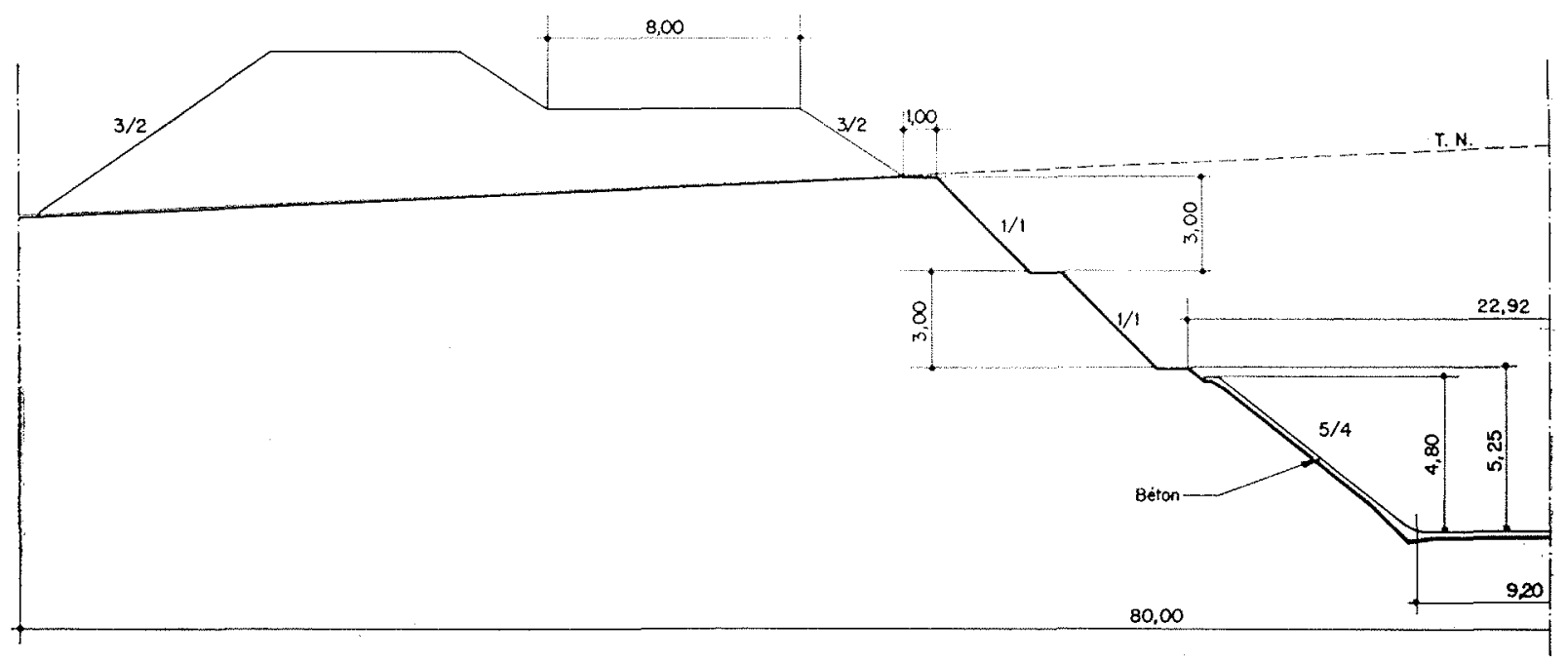

FIG. 7

Canal principal, de Pichegu à Saint-Gilles. Demi-profil en travers. Rive gauche.

normale des groupes électropompes dotés chacun d'un autotransformateur de démarrage).

Le poste sera relié à celui E.D.F. de Jonquières-Saint-Vincent par deux lignes $63.000 \mathrm{~V}$ autonomes.

La mise en roule et l'arêt sucessif des grou- pes seront normalement automatiques et asservis au niveau dans les biefs de restitution, qui devra etre maintenu constant, la station ne devant pas interrompre le réglage par l'aval de la ligne d'eau.

Après Pichegu, le canal principal, qui porte
Fig. 8. - Canal principal, tronçon Pichegu-Saint-Gilles. Terrassement de la cunette.

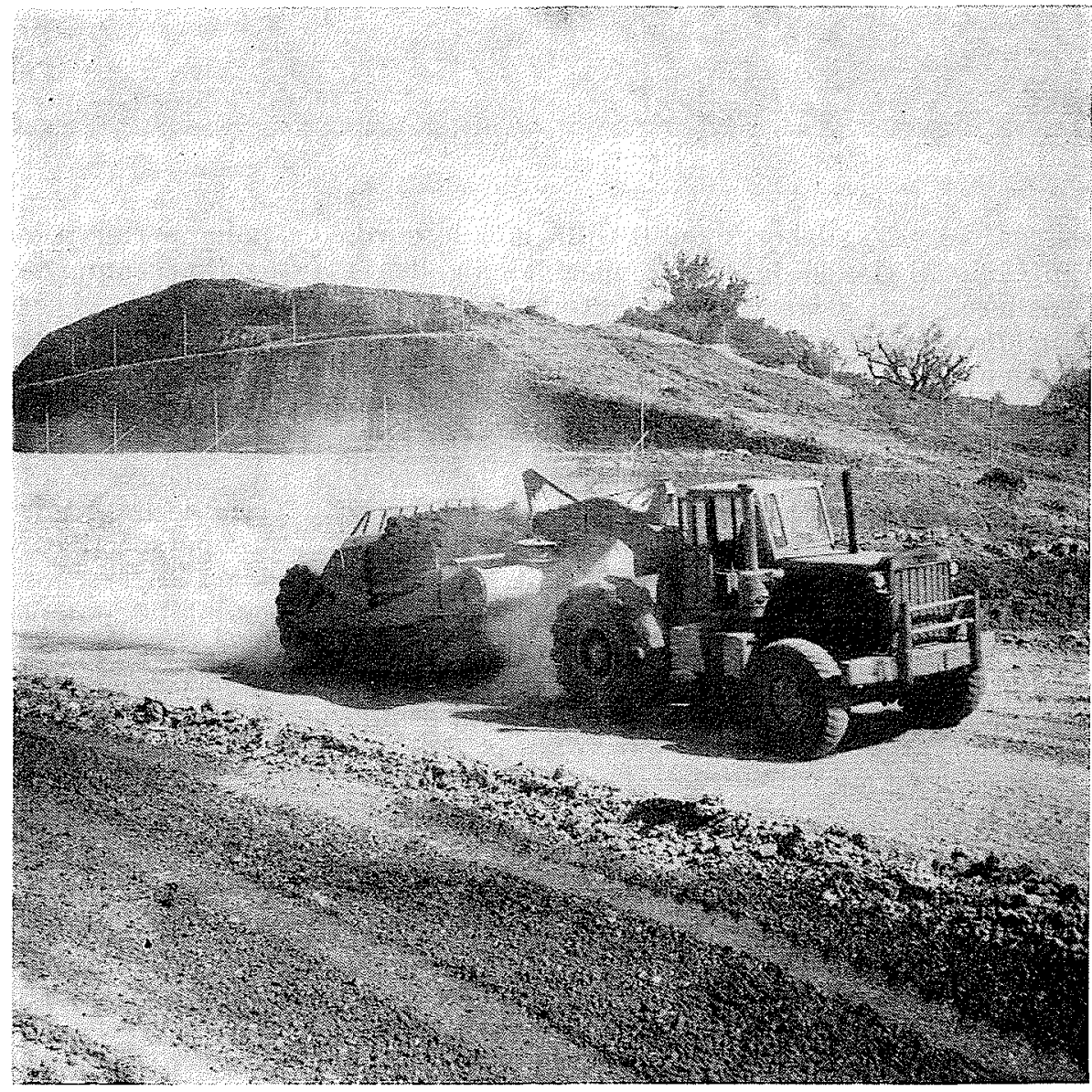




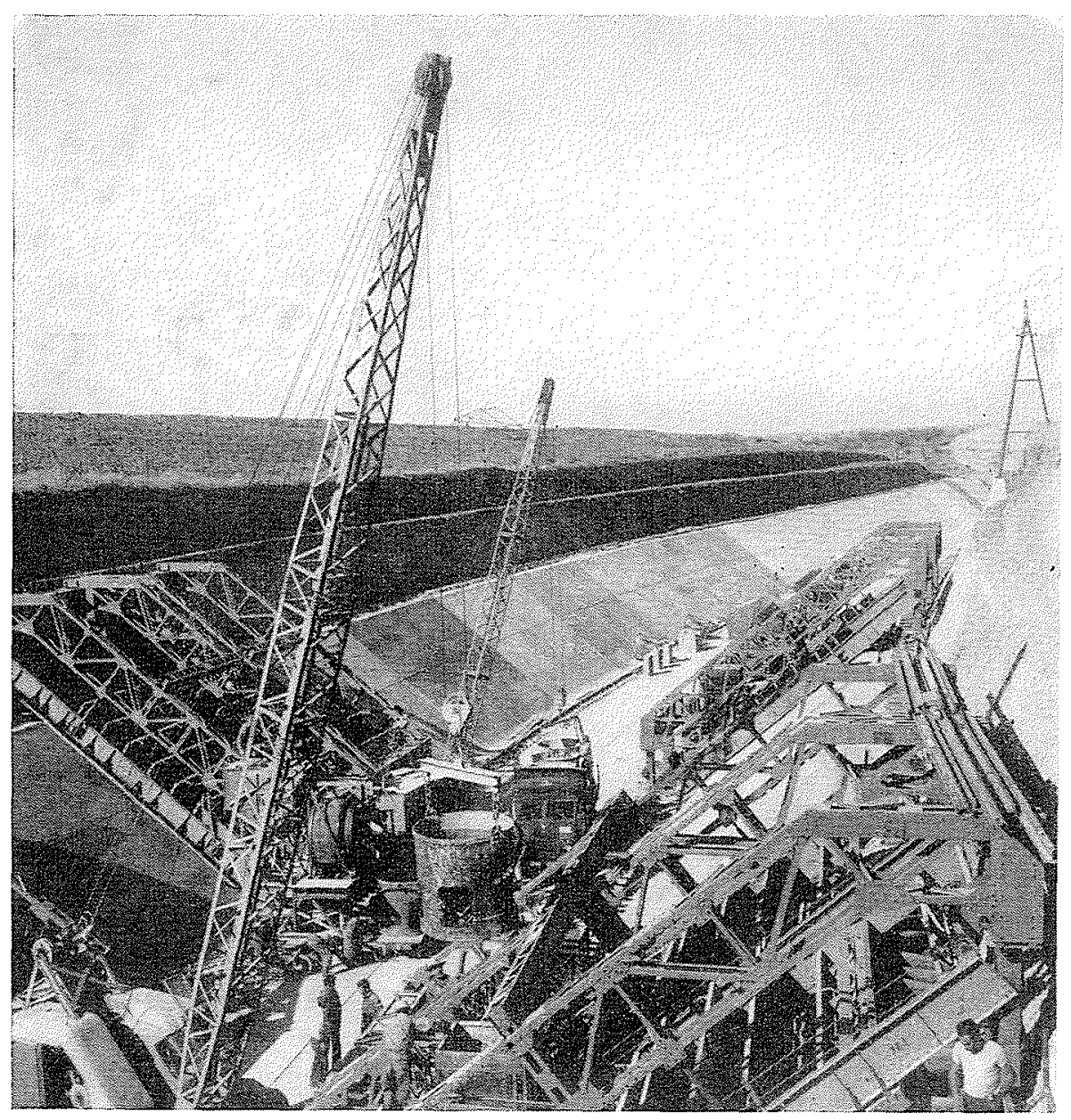

Fici. 9. - Canal principal, troncon Pichegu-Saint-Gilles. Revêtement des talus. encore $61 \mathrm{~m}^{3} / \mathrm{s}$, déborde Saint-Gilles par le nord, après avoir longé la lisière méridionale de la Costière, dont il traverse les promontoires par quelques tranchées assez importantes, la profondeur de certaines d'entre elles atteignant $14 \mathrm{~m}$.

Au-delà de Saint-Gilles, le tracé ne présente pas de difficultés particulières, sauf à la traversée des fleuves côtiers ou de certaines dépressions recoupées : Vistre, Vidourle, Lez, Mosson, Vène, Hérault, Tongue, Libron et dans le doublement de certains centres urbains, tel Montpellier. Il est jalonné par les centres de Vauvert, GrandGallargues, Lunel-Vieil, Mudaison, Montpellier, Fabrègue, Montbazin, Poussan, Loupian, Pinet, Nézignan-L'Evêque, Montblanc, Clairac, Cers et Béziers.

A l'aval de Montpellier, à $89 \mathrm{~km}$ de son origine au Rhône, la ligne d'eau du canal prineipal, dont la cote en ce point n'est plus que de $10,45 \mathrm{~m}$, doit être à nouveau relevée sur une hauteur de $31,50 \mathrm{~m}$. La station correspondante, située à la pointe nordest de la montagne de La Gardiole, qui borde la mer de Villeneuve-les-Maguelonne à Frontignan, relèvera le plan d'eau à la cote nécessaire $(40,19)$ pour permettre au canal de s'engager dans le couloir compris entre le flanc nord de La Gardiole et la lisière sud des garrigues de Pignan et de Cournonterral.

A la vérité, de La Gardiole à Loupian, le canal principal ne dessert qu'une bande de terres assez étroite et il est vraisemblable qu'on l'eùt arrèté a Villeneuve-les-Maguelonne si les hasses vallées de l'Hérault et de l'Orb n'avaient pas oflert les perspectives très favorables qu'elles présentent. Encore convient-il de limiter au minimum le débit à pomper à La Gardiole. A cet égard, le concours apporté par l'Orb est particulièrement précieux. Il en sera question plus loin, à propos de l'utilisation des fleuves còtiers et de l'aménagement de la zone ouest. Bornons-nous, ici, à indiquer que dans la conception actuelle, le canal principal issu du Rhône et qui devait initialement être prolongé au-delà de Béziers jusqu'à Coursan, où il aboutissait à l'Aude après avoir décrit une large boucle autour de l'étang de Capestang, sera arrêté en rive gauche de l'Orb.

Son tracé, entre Hérault et Orb, comportera d'ailleurs, entre le Libron et Montblanc, un court 
troncon à fond plat, qui permettra d'étendre, en année d'hydraulicité moyenne, jusqu'à la rive droite de l'Hérault, le bénéfice des irrigations gravitaires assurées par l'Orb, les eaux du Rhône, dont l'utilisation au-delà de La Gardiole exigent un pompage total sur $51,50 \mathrm{~m}$ n'étant plus appelées à franchir l'Hérault qu'en année sèche et seulement dans la mesure nécessaire pour compléter les débits en provenance de l'Orb. On évitera ainsi de surcroît, la traversée de l'Orb, qui dans le projet initial, comportait l'exécution d'un pont-bâche de $72 \mathrm{~m}$ de portée libre précédé et suivi d'aqueducs totalisant $2.380 \mathrm{~m}$ de longueur et relativement hauts au-dessus du fond de la vallée.

De son origine à Fourques jusqu'à Béziers, le canal principal du système d'irrigation, alimenté par le Rhône, aura $175 \mathrm{~km}$ de longueur.

Le canal principal ne domine qu'une partie du périmètre irrigable : 68.400 sur $128.900 \mathrm{ha}$, soit environ la moitié. Le complément, situé au nord de son tracé, est irrigué par pompage.

Ce complément s'anályse en un certain nombre de secteurs indépendants, dont le plus important est celui des Costières du Gard et de La Vistrenque.

\section{Périmètre des Costières et de la Vistrenque.}

L'ensemble du plateau des Costières du Gard et de la vallée du Vistre ou Vistrenque forme grossièrement un rectangle allongé du nord-est au sud-ouest, et constitue une zone de transition entre les garrigues calcaires du rebord sud-est des Cévennes et les basses plaines alluviales de la rive droite du Rhône. Les limites en sont les suivantes :

- Au nord-est, le rebord des Costières domine depuis Beaucaire jusqu'à Meynès la basse vallée du Gard.

- Au sud-est, le rebord du plateau est sensiblement parallèle au canal de navigation du Rhône à Sète depuis Beaucaire jusqu'à SaintGilles.

- Au sud-ouest, la limite de la zone à irriguer est jalonnée par les localités de Saint-Gilles, Générac, Vauvert et Uchaud.

- Au nord-ouest, cette limite est constituée par la R.N. 113 (Montpellier-Nîmes), depuis Uchaud jusqu'à Nîmes, puis par la R.N'. 86 (Nîmes-Avignon), depuis Nîmes jusqu'à la vallée du Gard, par Saint-Gervasy et Bezouce,

Ce secteur couvre une surface de 34.000 ha. Le débit d'irrigation nécessaire, soit $13,5 \mathrm{~m}^{3} / \mathrm{s}$ en pointe, prélevé dans le canal principal à Pichegu, à la cote 0,44 , est relevé par pompage d'une hauteur moyenne de $66,50 \mathrm{~m}$ et restitué à la cote 67 dans le canal primaire des Costières par deux conduites de $2,10 \mathrm{~m}$ de diamètre et de $700 \mathrm{~m}$ de longueur environ.

Ce canal primaire serpente d'abord sur $1,500 \mathrm{~km}$, sur le flanc sud-est du plateau des Costières, puis franchit par un souterrain de $500 \mathrm{~m}$ de longueur le rebord de celui-ci, pour déboucher sur le plateau. Il se développe ensuite du sudouest au nord-est jusqu'à la hauteur de Jonqières-Saint-Vincent, où il se termine à la cote 63,50 .

Réserve faite du pompage de tête, l'ensemble du périmètre est irrigable par gravité, sans autre pompage, à l'exception :

- Des terres hautes du plateau de GaronsGénérac, soit environ 7.600 ha, pour lesquelles il faudra relever un débit de $3 \mathrm{~m}^{3} / \mathrm{s}$ en pointe, de la cote 66 à la cote 91 .

-- Des terres hautes du plateau de Meynès, soit environ 3.500 ha, pour lesquelles il faudra relever un débit de $1.800 \mathrm{l} / \mathrm{s}$ de la cote 63,5 à la cote 75 .

Ceci n'exclut pas la réalisation de pompages supplémentaires destinés à assurer la mise en charge d'une partie des eaux d'irrigation utilisées alors par aspersion.

Les caractéristiques du canal primaire, entièrement bétonné (revêtement non armé de $0,10 \mathrm{~m}$ d'épaisseur), sont rassemblées ci-dessous.

\begin{tabular}{|c|c|c|c|c|c|}
\hline P.K. & $\begin{array}{l}\text { Longueurs } \\
\text { km }\end{array}$ & $\begin{array}{c}\text { Débits } \\
\mathrm{m}^{3} / \mathrm{s}\end{array}$ & $\begin{array}{l}\text { Largeur } \\
\text { au radier }\end{array}$ & Talus & Pentes \\
\hline 0 & 5,1 & 13,5 & 3 & $3 / 2$ & $12.10-5$ \\
\hline 0,100 & 3,1 & 10,5 & 3 & $3 / 2$ & $12.10^{-5}$ \\
\hline 0,200 & 3,6 & 5,8 & 2 & $3 / 2$ & $15.10^{-5}$ \\
\hline 11,000 & 4 & 4 & 2 & $3 / 2$ & $15.10^{-5}$ \\
\hline 18.800 & 2,8 & 2,5 & 1 & $3 / 2$ & $15.10^{-} \overline{5}$ \\
\hline
\end{tabular}

Le canal sera d'un bout à l'autre en commande par l'aval, les différentes stations de pompage étant elles-mêmes en commande par l'aval.

\section{Secteur Basse-Vistrenque-La Vaunage.}

Il intéresse la rive droite de la Basse-Vistrenque (Vestric, Codognan, Vergèze) et la cuvette de Calvisson, Clarensac, Saint-Còme, Caveirac, située derrière la dernière ride des garrigues.

Les coteaux de Villevieille, Fontanès, Lecques, dans le cours moyen du Vidourle, seront également desservis par ce réseau.

Le débit total nécessaire, soit $3,8 \mathrm{~m}^{3} / \mathrm{s}$, sera 
prélevé dans le canal principal au niveau de Grand-Gallargues par une station de pompage à deux étages :

$1^{\text {or }}$ étage : $1,400 \mathrm{~m}^{3} / \mathrm{s}$, sous une hauteur de $4 \mathrm{~m}$, destiné à la Basse-Vistrenque.

$2^{\circ}$ étage : $2,400 \mathrm{~m}^{3} / \mathrm{s}$, sous une hauteur totale de $30 \mathrm{~m}$.

Ce dernier débit transitera par un canal vers Calvisson, d'où un réseau de canalisations le répartira sur le périmètre desservi.

Des stations de pompage de faible importance permettront de dominer les parties surélevées : ver's Aujargues, Fontanès...

La superficie desservie par la station de GrandGallargues atteint 8.600 hectares, dont 5.600 seront effectivement mis à l'irrigation.

\section{Secteur de Lunel-Viel.}

Il couvre la région située au-dessus du canal principal, entre le Vidourle et Montpellier. Il comprend les coteaux de Saint-Geniès-des-Mourgues, de Castries, Sommières, Aspères, Lecques, d'une part, et la plaine bordant, au sud, les garrigues de Saint-Brès et de Baillargues, d'autre part.

La station de pompage desservant ce secteur prélèvera $6,5 \mathrm{~m}^{3} / \mathrm{s}$ sur le canal principal, à $2 \mathrm{~km}$ au sud-ouest de Lunel-Viel, au P.K. 60, où la cote du plan d'eau est 14,21. La hauteur de refoulement prévue est de $13 \mathrm{~m}$.

Le débit refoulé est réparti entre deux branches d'irrigation, la branche basse qui se développe, sur $13 \mathrm{~km}$, entre les cotes 27,00 et 22,00 et passe à Baillargues et Saint-Aunès, et la bran. che haute, sensiblement parallèle à la première, mais décalée en hauteur de $20 \mathrm{~m}$ moyennant repompage de la cote 26,00 à la cote 46,00 . Cette deuxième branche, longue de $20 \mathrm{~km}$, passe à proximité des villages de Saint-Geniès-des-Mourgues et de Vendargues et se termine à Castelnaule-Lez. Une autre antenne remonte la vallée du Vidourle ver's Campagne, Aspères, Lecques.

La surface desservie par le réseau de LunelViel couvre 14.600 ha, dont 9.500 pourront être effectivement irrigués.

\section{Secteur de Mireval-Sète.}

A proximité de Villeneuve-les-Maguelonne, au P.K. 89 du canal principal, il est prévu de dériver de celui-ci un débit de $2,0 \mathrm{~m}^{3} / \mathrm{s}$.

Ce débit est destiné, d'une part à irriguer les plaines littorales situées au pied de la montagne de La Gardiole, entre Mireval et Frontignan, d'autre part à apporter à la ville de Sète et à la région bordière du bassin de Thau l'eau nécessaire à leur développement industriel très prometteur. Le débit réservé à cette fin serait de l'ordre de $1 \mathrm{~m}^{3} / \mathrm{s}$. La branche Mireval-Sète aurait $7.400 \mathrm{~m}$ de longueur. La surface desservie serait de 1.600 ha.

\section{Secteur de Fabrègues.}

Le réseau de Fabrègues irriguera la région comprise entre la montagne de La Gardiole et les garrigues qui prolongent vers le nord-ouest la montagne de la Moure.

Le réseau sera alimenté, à partir du canal principal, par un tronc commun de $7.100 \mathrm{~m}$ de longueur traversant la plaine de Fabrègues à Cournonterral.

Ce canal répartira un débit de $1,5 \mathrm{~m}^{3} / \mathrm{s}$ entre trois branches. L'une se dirigera, par gravité, vers Laverune; les deux autres, après repompage à la cote 57, irrigueront les parties hautes de la plaine, respectivement vers Juvignac et Montbazin.

La surface totale couverte par le réseau de Fabrègues sera de 6.300 ha.

Compte non tenu de celles éventuellement nécessaires pour mettre en charge certains réseaux ou parties de réseau orientés vers l'irrigation par aspersion, les stations de pompage dont l'équipement est actuellement prévu sur le périmètre est, tant pour remonter la ligne d'eau du canal principal que pour alimenter les branches maîtresses dérivées, sont indiquées dans le tableau suivant :

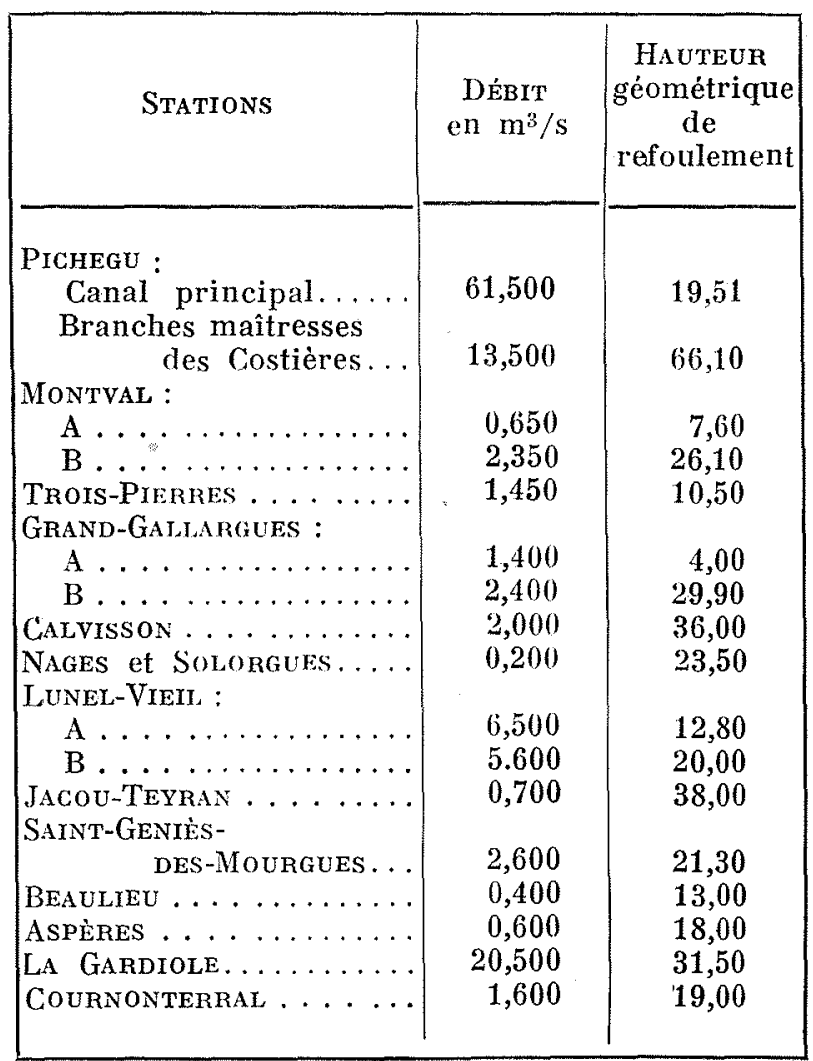




\section{LE SYSTEME D'IRRIGATION DE LA ZONE OUEST}

Pour éviter d'inutiles transports et des frais de pompage onéreux, l'eau du Rhône étant prise à une cote assez basse $(+1,10$ N.G.F. $)$, il était normal de chercher à tirer parti des cours d'eau recoupés par le canal principal dans son développement vers l'ouest.

Les cours d'eau sont malheureusement relativement peu nombreux. De surcroût, leur régime méditerranéen caractérisé par des étiages d'été très marqués est mal adapté aux besoins agricoles. Enfin, les caractéristiques topographiques ou géologiques de leur vallée ne se prêtent qu'exceptionnellement à la construction de réservoirs d'accumulation.

Les seuls fleuves notables recoupés d'est en ouest sont: le Vidourle, l'Hérault, l'Orb et, en limite de la zone d'action de la Compagnie, l'Aude.

\section{Le Vidourle.}

Le Vidourle, qui prend sa source au Liron, à $1.180 \mathrm{~m}$ d'altitude, et dont la pente génćrale de son origine à son entrée ẹ plaine, à Sommières, est asse forte, est tristement conmu pour ses crues subites et violentes : les «Vidourlades». Il est asez paradoxal qu'en dépit des graves mófaits commis par lui de temps immémorial, tant au préjudice des bourgades qu'il traverse (Sauve, Quissac, Sommières) que des plaines basses qu'il inonde au sud de Grand-Gallargues, ce fleuve n'ait été, jusqu'à ces temps derniers, l'objet d'aucun jaugeage. Seules des échelles de crue ont été

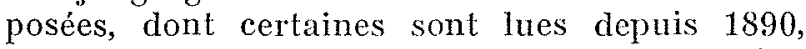
qui permettent de mesurer et, dans une certaine mesure, d'annoncer les crues.

La Compagnie a entrepris une étude hydrologique systématique du Vidourle et installé à cet effet, tant sur le fleuve lui-même que sur ses principaux affluents (Crieulon, Rieumassel), quelques échelles limnigraphiques et quelques stations de jaugeage, dont une permanente, du type téléphérique, à Sommières.

Elle l'a fait essentiellement pour concourir à l'étude des crues du fleuve et à la recherche des moyens propres à les prévenir, mais non dans l'espoir de pouvoir tirer un parti utile du Vidourle pour l'alimentation de son réseau d'irrigation. Aucune possibilité n'existe, en effet, malheureusement, de constituer des réserves saisonnières sur le cours de ce fleuve. Dans la partie du bassin, où topographiquement quelcues sites, d'ailleurs médiocres, peuvent être trouvés, les terrains intéressés par les retenues sont karstiques et n'autorisent aucun espoir d'emmagasinement durable.
Au reste, la capacité des quelques barrages dont la construction peut être envisagée sur ie Vidourle, le Crieulon et le Rieumassel, suffirait à peine à écrêter à $1.000 \mathrm{~m}^{3} / \mathrm{s}$, chiffre à la rigueur admissible, les crues qui, à l'entrée en plaine, atteignent maintenant $1.600 \mathrm{~m}^{3} / \mathrm{s}$.

Ces barrages, qu'il faudra bien réaliser un jour pour protéger les populations de la moyenne vallée et les riches plaines du cours inférieur contre les inondations, ne sauraient donc être d'aucun secours pour l'irrigation.

\section{L'Hérault.}

Pour l'Hérault, les conclusions sont à peu près les mêmes.

Le seul barrage important réalisable est celui de Saint-Guilhem-le-Désert, à $10 \mathrm{~km}$ en amont de Gignac.

Ce barrage, dont les terrains d'assise et de cuvette sont les calcaires et dolomies très caverneux et fissurés du Jurassique moyen et supérieur, ne saurait servir de base à un projet d'irrigation. Sa construction paraît néanmoins désirable pour protéger lá vallée de l'Hérault, à l'aval de Gignac, contre les débordements du fleuve, les crues de printemps favorisant, par l'humidité qu'elles entraînent, le développement des maladies cryptogamiques de la vigne (mildiou), et les crues d'automne entraînant la ruine des récoltes pendantes.

D'ailleurs, s'il s'avérait à l'expérience, le barrage étant supposé construit aux fins indiquées ci-dessus, que l'étanchéité de la cuvette soit moins mauvaise qu'on ne le pense et autorise techniquement le report sur l'été d'une partie des débits d'hiver, ce report ne pourrait pratiquement se faire qu'au préjudice de la protection contre les inondations.

Un arbitrage entre la fonction d'écrêtement des crues et celle d'irrigation deviendrait nécessaire, option d'autant plus délicate à faire que, limitée à 144 millions de mètres cubes (pour la cote de retenue maximum 125 N.G.F. correspondant à une hauteur au-dessus de l'étiage de $50 \mathrm{~m}$ ) par l'existence, à son amont, du village de Saint-Bauzille-du-Putois, la retenue de SaintGuilhem n'assurera des plus grosses crues connues qu'un écrêtement imparfait dans la basse vallée de l'Hérault, compte tenu de l'importance des débits incontrôlés apportés à son aval par certains gros affluents (la Lergue en particulier). Cet état de choses est évidemment regrettable, car la partie de la plaine de l'Hérault située entre Gignac et Pézenas, au nord du canal princi- 
pal issu du Rhône et que celui-ci ne dominera pas, est très étendue et fort belle.

Indiquons, pour mémoire, qu'avant la création de l'E.D.F., le projet avait été élaboré par une des sociétés productrices régionales, de dériver dans l'Hérault une partie des eaux de la Dourbie, qui ressortit au bassin du Tarn. Les eaux correspondantes, régularisées par un barrage de 39 millions de $\mathrm{m}^{3}$ sur le Linguas, auraient été turbinées en deux chutes, l'une à Salagosse, l'autre au Vigan sur $1.000 \mathrm{~m}$ de hauteur totale. Cet aménagement - outre qu'il n'aurait sans doute, s'il avait été réalisé, apporté d'eau dans l'Hérault que durant les mois où les besoins en puis. sance sont les plus grands, c'est-à-dire à contresaison par rapport aux besoins agricoles - a maintenant cédé le pas à un projet exhaustif dans lequel les eaux des rivières drainant le flanc nord de l'Aigoual se conjuguent avec celles du Haut-Tarn, pour être dérivées finalement dans le système des Gardons après avoir franchi la chaîne des Cévennes.

Ainsi, pour n'être pas absolument nul, le parti que l'on peut espérer tirer des eaux de l'Hérault pour l'irrigation du Bas-Languedoc est bien mince et trop aléatoire pour justifier seul un aménagement dispendieux. Tout au plus peuton envisager avec certitude d'intégrer, moyennant un pompage de hauteur modeste, les eaux sauvages de l'Hérault disponibles en dehors de l'étiage dans le canal principal issu du Rhône, au point où ce canal franchira le fleuve. Etant donné le caractère aléatoire de ces apports et leur quasi-nullité au moment de la pointe d'irrigation d'été, aucune réduction ne résultera de cette intégration, ni du gabarit du canal principal, ni de la puissance installée dans les stations de pompage qui en relèvent la ligne d'eau à Pichegu et à La Gardiole, mais seulement une diminution des quantités d'eau dérivées du Rhône et par conséquent une économie sur les dépenses d'énergie de pompage dans les deux stations précitées.

\section{L'Orb.}

L'Orb offre par contre d'intéressantes possibilités. Si son débit d'étiage est faible (il peut descendre à $500 \mathrm{l} / \mathrm{s}$ environ), ses apports totaux annuels sont importants (de l'ordre de $683 \mathrm{mil}$ lions de $\mathrm{m}^{3}$ en année moyenne).

Ce fleuve est d'autre part connu pour la violence et l'importance de ses crues, dont les villes de la basse-vallée et Béziers en particulier sont souvent les victimes. La crue de 1953 , une des plus fortes connues, paraît avoir atteint $2.900 \mathrm{~m}^{3} / \mathrm{s}$ à Béziers et l'on peut estimer qu'au moins une fois tous les deux ans les bas quartiers de cette ville sont inondés.

D'autre part, l'E.D.F. est sur le point d'entre- prendre l'aménagement de Montahut qui entraînera la dérivation dans le Jaur, affluent de rive droite de l'Orb, pour être turbinées sous $600 \mathrm{~m}$ de chute, d'une partie des eaux de l'Agout et de la Vesbre, rivières qui ressortissent au bassin du Tarn. Cette dérivation portera, en année moyenne, sur 183 millions de $\mathrm{m}^{3}$ d'eau, partiellement régularisés par une retenue de 40 millions de $\mathrm{m}^{3}$, à construire à Laouzas sur la Vesbre.

Malheureusement pour l'agriculture, cet apport très substantiel interviendra d'octobre à mai. Il accusera encore la discordance entre le calendrier des ressources hydrauliques disponibles et celui des besoins à satisfaire. Mais la vallée de l'Orb, contrairement à celles du Vidourle et de l'Hérault, se prête à l'aménagement de quelques barrages de retenue, en sorte qu'il devient possible de tirer, dans des conditions économiques acceptables, un parti utile des débits sauvages de l'Orb et des apports complémentaires en provenance du bassin du rarn.

Plusieurs sites ont été reconnus, dont trois ont déjà fait l'objet d'une étude.

\section{Barrage du Rieuberlou.}

L'un d'eux intéresse un petit affuent de l'Orb, le Rieuberlou, qui rejoint le fleuve à quelques kilomètres en aval du village de Roquebrun. Le Rieuberlou n'a par lui-même qu'un débit très faible, dont on est seulement en droit de compter qu'il compensera largement les pertes de la retenue durant le cycle d'exploitation de celle-ci. La cuvette devra donc être remplie par dérivation de l'Orb. Celui-ci n'est séparé de la vallée de son affluent que par une ride montagneuse assez étroite. Dans un premier temps l'eau sera prélevée dans l'Orb par pompage.

Le barrage de prise, dont la construction est prévue sur l'Orb à Lugné, à quelques centaines de mètres en amont du confluent avec le Rieuberlou et qui commandera en rive gauche la prise d'eau du canal trone commun d'irrigation, assurera en rive droite la dérivation des eaux nécessaires dans un canal utilisant partiellement le cours inférieur du Rieuberlou jusqu'au pied aval du barrage d'accumulation où sera installée la station de pompage provisoire.

Le barrage de prise de Lugné comportera, entre la cote du seuil fixe déversant $(65,00)$ et le sommet de la bouchure mobile dont il sera équipé $(68,00)$, une capacité de 1 million de $\mathbf{m}^{3}$. Celle-ci servira de réserve compensatrice des lâchures de l'usine de Montahut, appelée à faire des pointes de $18 \mathrm{~m}^{3} / \mathrm{s}$ pour des éclusées journalières de l'ordre de $1.200 .000 \mathrm{~m}^{3}$. Elle permettra donc de réduire sensiblement l'équipement de l'usine de pompage qui pourra, sans inconvénient, être limité à trois groupes de $2.5001 / \mathrm{s}$. 
Ces groupes fonctionneront sous une charge variable, en fonction de l'état de la réserve, depuis un minimum technique d'une trentaine de mètres jusqu'à un maximum de $65 \mathrm{~m}$. Il est à noter que ces groupes trouveront ultérieurement une rentabilité logique dans une station de pompage qu'il est prévu d'installer à Murviel, sur l'une des artères maîtresses du réseau d'irrigation de rive gauche, pour desservir une dizaine de milliers d'hectares dans la région de Servian.

Par la suite, la réserve du Rieuberlou sera remplie par une dérivation gravitaire des eaux de l'Orb, que rendra possible la construction sur ce fleuve, à l'amont immédiat du village de Ceps, d'un autre barrage d'accumulation dont nous parlerons plus loin. La liaison Ceps-Rieuberlou sera alors assurée par une galerie de $3.900 \mathrm{~m}$ de longueur et de $4,30 \mathrm{~m}$ de diamètre intérieur.

Comme nous le verrons plus loin, la possibilité de construire le barrage de Ceps, tout au moins celle de réaliser en ce point un barrage utile pour l'irrigation, n'est pas encore absolument démontrée, compte tenu des caractéristiques géologiques de la cuvette. Cette construction s'avérerait-elle impossible ou économiquement inopportune, que le remplissage gravitaire du Rieuberlou n'en serait pas moins réalisable en reprenant le projet initialement élaboré d'une galerie amenant dans le réservoir les eaux du Jaur grossies des apports du bassin du Tarn et dérivées, par un barrage de prise de faible hauteur, peu à l'aval de la restitution de la centrale de Montahut. Mais, tenu compte du prix auquel l'énergie peut nous être fournie en heures creuses et des facilités de production inhérentes à l'équipement de quelques chutes connexes des aménagements hydro-agricoles projetés et sur lesquelles nous reviendrons, il semble que cette liaison gravitaire, qui nécessiterait la construction d'une galerie de section minimum de $7.700 \mathrm{~m}$ de longueur, soit économiquement moins avantageuse que le maintien en service de la station de pompage provisoire.

Le barrage du Rieuberlou aura $60 \mathrm{~m}$ de hauteur totale et $280 \mathrm{~m}$ de développement en crête. A la cote 130 , qui est celle de la retenue normale, sa capacité sera de 44,3 millions de $\mathrm{m}^{3}$. II est prévu en enrochements avec masque épais d'étanchéité en terre incliné. L'hétérogénéité du terrain de fondation (bancs alternés de schistes siluriens et de quartzites subverticaux transversaux à la vallée) et les qualités mécaniques assez médiocres des bancs de schistes ont conduit à écarter les types de barrages rigides. La cuvette, essentiellement schisteuse, est à coup sûr étanche.

\section{Barrage de Ceps.}

A quelques centaines de mètres en amont du village de Ceps, la vallée de l'Orb traverse un massif schisteux primaire étanche et de qualités mécaniques acceptables. Elle se prête assez bien à l'édification d'un barrage qui sera vraisemblablement du type poids évidé.

L'ouvrage aurait $320 \mathrm{~m}$ de longueur, environ, et $70 \mathrm{~m}$ de hauteur. A la cote de la retenue normale (147) le volume emmagasiné serait de 98 millions de $\mathrm{m}^{3}$. L'ouvrage serait entièrement fondé sur les schistes. Malheureusement, des bancs calcaires sont baignés par la cuvette, que l'on retrouve à l'aval du barrage, où ils affleurent dans une vallée affluente à une cote très sensiblement inférieure à celle de la retenue maximum.

La longueur du shunt ainsi susceptible de s'établir par les calcaires est seulement de l'ordre de $2.000 \mathrm{~m}$ et il est à craindre, à raison du caractère assez karstique de ces formations, que les pertes de charge ne soient pas telles que les débits de fuite restent compris dans des limites acceptables. Des études complémentaires ont été entreprises pour s'en assurer.

A la vérité, deux circonstances favorables jouent en faveur de l'aménagement.

D'une part, les apports naturels de l'Orb, compte tenu de ceux supplémentaires à provenir de la dérivation de Montahut, sont assez imporlants jusqu'en fin mai pour que le remplissage définitif de la retenue puisse, sans inconvénient, ètre reculé jusqu'à cette époque. Comme, d'autre part, le déstockage pour les besoins agricoles aura lieu en juillet et août, on voit que la durée du report saisonnier sera relativement courte.

Par ailleurs, il faudra, en toute saison, Iaisser un certain débit dans l'Orb, pour la satisfaction des besoins autres que d'irrigation, ne fut-ce que pour des raisons d'hygiène. Enfìn et surtout, les arrosages devant commencer en avril, il sera, dès cette époque, nécessaire de lâcher dans l'Orb, pour être intégrés à Lugné dans le canal adducteur du système d'irrigation, des débits qui marqueront, en principe, l'évolution suivante :

\begin{tabular}{|c|c|c|c|c|c|c|}
\hline Mois & Avril & Mai & Juin & Juill. & Août & Sept. \\
\hline $\begin{array}{c}\text { Débits } \\
\text { en } \\
\mathrm{m}^{3} / \mathrm{s}\end{array}$ & 4 & 12,7 & 19,6 & 38,5 & 28 & 6 \\
\hline
\end{tabular}

Ainsi, il faudrait que les débits de fuite du barrage soient énormes pour que, dépassant ceux nécessaires à l'aval, ils constituent de véritables pertes. On a donc quelque raison d'espérer que les études complémentaires en cours permettront de retenir Ceps dans la liste des barrages suscep- 
tibles d'assurer pour l'irrigation une régularisation saisonnière de l'Orb.

Cet ouvrage aurait, par ailleurs, - et à cet égard sa convenance est d'ores et déjà certaine, - un très gros intérêt pour la protection des riverains d'aval contre les inondations. Il est peu courant qu'un barrage puisse, à la fois, servir à l'accumulation saisonnière et à l'écrêtement des crues, à moins que sa capacité ne soit énorme et qu'une tranche particulière puisse être affectée à chacune de ces deux fonctions sans préjudice pour lautre.

Ici, à la vérité, la capacité totale du barrage $\left(98 \mathrm{hm}^{3}\right)$ n'est pas superfétatoire au regard des besoins agricoles et, par conséquent, si les deux fonctions précitées devaient être simultanément remplies, l'écrêtement des crues ne pourrait être réalisé qu'au prix d'une réduction des débits disponibles pour l'irrigation. Mais il se trouve que ces deux fonctions peuvent être successivement assurées. Comme nous l'avons signalé, en effet, les débits de printemps, compte tenu des apports de Montahut, seront normalement assez abondants pour que, sans préjudice pour son remplissage final, la retenue de Ceps puisse, d'octobre à mars, être maintenue quasiment vide et, par conséquent entièrement disponible pour l'écrêtement des crues. Orr, la presque totalité de celles actuellement dommageables survient précisément durant cette période. On relève bien quelques crues d'avril assez importantes, mais elles sont fort rares et localisées dans la première quinzaine du mois. A la vérité, une crue catastrophique comme celle de 1953 - d'ailleurs composée de deux ondes, dont les maxima espacés de 46 heures ont atteint à Béziers les cotes N.G.F. 12,33 et 11,98, correspondant à des débits de pointe de 2.900 et $2.500 \mathrm{~m}^{3} / \mathrm{s}$ environ - n'aurait été que partiellement écrêtée. Elle aurait encore été de l'ordre de $1.400 \mathrm{~m}^{3} / \mathrm{s}$, mais il s'agit là d'une crue centenaire et les dégâts occasionnés par une crue de $1.400 \mathrm{~m}^{3} / \mathrm{s}$, pour n'être pas négligeables, ne sont cependant pas absolument catastrophiques.

\section{Barrage d'Avène-Ceilhes.}

Ce barrage se situerait sensiblement plus en amont sur le cours de l'Orb. Il s'implanterait dans un défilé assez resserré, dont le profil et la qualité du sol de fondation (grès schisteux) justifient pleinement l'adoption d'une voûte mince.

L'ouvrage aurait $63 \mathrm{~m}$ de hauteur au-dessus des fondations et $230 \mathrm{~m}$ de développement en crête. La capacité de retenue normale serait de 33,6 millions de $\mathrm{m}^{3}$. Bien que situé assez haut sur le bassin versant (la surface de celui-ci n'est plus en ce point que de $125 \mathrm{~km}^{2}$ ), son remplissage annuel ne pose aucun problème. L'étanchéité de la cuvette est par ailleurs assurée, bien que les calcaires y affleurent, le banc correspondant ne réapparaissant à l'aval du barrage qu'à une cote supérieure à celle de la retenue.

La capacité cumulée des trois barrages dont nons venons de parler atteindrait donc 175,9 millions de $\mathrm{m}^{3}$. Compte tenu de la contribution possible de l'Aude, dont il sera question plus loin, cette capacité régularisatrice des apports de l'Orb permettrait d'irriguer à partir de ce fleuve, en année d'hydraulicité déficitaire, type 1924, 79.000 hectares, le débit maximum à dériver de l'Orb atteignant alors (en juillet) : $38,50 \mathrm{~m}^{3} / \mathrm{s}$.

L'année 1924 ne saurait être considérée comme étant la plus mauvaise. En fait, elle correspond - autant qu'on en puisse juger eu égard au nombre assez limité de campagnes d'observation dont on dispose - à une année ne laissant derrière elle que $15 \%$ environ d'années d'hydraulicité moindre, mais c'en̂t été faire preuve de malthusianisme que d'adopter pour ce faire l'année la plus mauvaise. Des restrictions peuvent, sans grand inconvénient, être apportées à certaines irrigations, sous réserve qu'elles restent exceptionnelles et comprises dans certaines limites. Au reste, dans le cas particulier, il n'y aura pas de césure absolue entre le réseau tributaire du Rhône et celui desservi par le complexe OrbAude.

Entre Hérault el Orb, il est prévu, qu’à la charnière des deux systèmes, certains échanges seront possibles, les eaux du Rhône venant, notamment, relayer celles de l'Orb, en année d'hydraulicité particulièrement déficitaire, dans l'irrigation d'une douzaine de milliers d'hectares.

Il est à noter, d'autre part, que les aménagements prévus sur l'Orb à des fins purement agricoles, auront comme conséquence secondaire, mais très heureuse, de rendre possible et rentable l'équipement d'un certain nombre de chutes :

D'une part, la chute créée par le barrage de Ceilhes-Avène permettra de turbiner, soit en pied de barrage, soit dans la pointe amont du remous de l'ouvrage de prise à la centrale du Truscas qui lui fait suite, les eaux sauvages de l'Orb affluant à Ceilhes.

D’autre part, sans préjudice pour le rôle d'écrêteur de crues qu'il aura à remplir d'octobre à mars, le barrage de Ceps pourra être mainlenu durant cette période à la cote 110 (à laquelle ne correspond qu'une réserve morte de $\left.9,8 \mathrm{hm}^{3}\right)$. Les débits sauvages affluant durant cette période (et ultérieurement ceux provenant du déstockage des retenues) pourront donc être turbinés à la cote 65 dans le plan d'eau du barrage de prise de Iugné après transit par le réservoir du Rieuberlou.

Enfin, le canal tronc commun du système d'irrigation qui, avant de quitter la vallée, se développe sur une dizaine de kilomètres au long du fleuve, dominera celui-ci de $38 \mathrm{~m}$ à la sortie de 
la cluse de Réals. Ce canal ne devant être utilisé pour l'irrigation que d'avril à fin septembre, pourra servir de canal d'amenée à une centrale au fil de l'eau, utilisant cette dénivelée de $38 \mathrm{~m}$, pendant toute la période complémentaire qui coïncide précisément avec celle durant laquelle les débits disponibles sont les plus abondants et les besoins en énergie les plus intenses.

Les caractéristiques de ces diverses centrales, dont l'étude de détail reste à faire, seraient approximativement les suivantes :

\begin{tabular}{|c|c|c|c|}
\hline \multirow{2}{*}{ Caractíristiques } & \multicolumn{3}{|c|}{ Centrales de } \\
\hline & AVÉNE & LUGNÉ & RÉals \\
\hline Hauteur de chute maximum $(\mathrm{m})$.. & 86 & 64,50 & 38 \\
\hline $\begin{array}{l}\text { Débit maximum d'équipement } \\
\quad\left(\mathrm{m}^{3} / \mathrm{s}\right) \ldots \ldots \ldots \ldots \ldots \ldots \ldots \ldots\end{array}$ & 17 & 60 & 38,5 \\
\hline $\begin{array}{c}\text { Energie productible en année } \\
\text { moyenne }\left(10^{6} \mathrm{kWh}\right) \ldots \ldots \ldots\end{array}$ & 19 & 78 & 42 \\
\hline
\end{tabular}

Il est a noter que le productible indiqué pour l'usine d'Avène est à coup sûr grossièrement sous-estimé. Les apports ont, en effet, été calculés en fonction des observations faites par l'E.D.F. à la prise de l'usine du Truscas. Or, ces observations ne portent que sur les débits inférieurs à $18 \mathrm{~m}^{3} / \mathrm{s}$. Il est donc certain, d'une part, que le barrage d'Avène-Ceilhes sera entièrement rempli bien avant la fin de l'hiver et, d'autre part, que les apports turbinables sous chute normale à partir de ce moment, seront beaucoup plus importants qu'il n'a été estimé.

Il appartiendra à l'E.D.F. d'équiper ces diverses centrales, sauf à elle à verser à la Compagnie une redevance d'usage des équipements généraux (barrage d'accumulation ou de dérivation, canaux adducteurs) réalisés par celle-ci et utilisés subsidiairement pour la production d'énergie électrique.

\section{L'Aude.}

Le régime de l'Aude, fleuve pyrénéen par son haut bassin, est légèrement différent de celui de l'Orb, issu des contreforts orientaux de la Montagne Noire, et, de ce fait, l'intégration de ce fleuve dans un complexe avec l'Orb est particuliè- rement intéressante. Il n'en reste que les étiages sont malheureusement sensiblement concomitants et que l'Aude, comme l'Orb, nécessite le report sur l'été d'une partie des débits d'hiver. Une tranche agricole de 2,5 millions de $\mathrm{m}^{3}$ existe sur la retenue de Puyvalador et une nouvelle tranche de $7.500 .000 \mathrm{~m}^{3}$ est prévue sur le barrage de Matémale, actuellement en cours de construction par l'E.D.F. Il a été admis que la Compagnie pourrait, sous réserve du respect des droits acquis, utiliser les eaux de l'Aude actuellement disponibles à la limite de sa zone d'action dans le Narbonnais, compte tenu des réserves précitées sur Puyvalador et Matémale, mais sous condition de ménager l'alimentation rationnelle de 10.000 ha de terres à irriguer plus en amont de sa zone d'action, notamment dans le Minervois.

Les eaux de l'Aude seront intégrées dans le réseau d'irrigation du périmètre ouest, partie par gravité, partie par pompage.

La seule branche de ce réseau susceptible d'être alimentée gravitairement par l'Aude est celle constituée par la Robine de Narbonne qui dessert 6.800 ha de terres entre Moussoulens et l'étang du Sigean. Un autre secteur de 3.200 ha dans la région de Cuxac-Coursan pourra être atteint, moyennant un pompage de hauteur extrêmement faible à l'origine du canal d'atterrissement de Capestang ( 2 à $3 \mathrm{~m}$ au maximum). Les autres secteurs sur lesquels, en année sèche, il conviendra d'introduire les eaux sauvages disponibles sur l'Aude pour ménager aussi longtemps que possible les eaux accumulées dans les réservoirs de l'Orb, nécessiteront des pompages de hauteur plus importante : $15 \mathrm{~m}$ pour l'irrigation de 2.800 ha dans la région d'Argens et de Canet, $33 \mathrm{~m}$ pour l'irrigation de 10.000 ha répartis par moitié entre la rive droite de l'Aude (région de Villedaigne, Ornaisons, Cruscades, Lézignan) et la rive gauche (secteur compris entre Ventenac et le château de Seriège).

A noter, d'ailleurs, qu'en année d'hydraulicité moyenne, les eaux de l'Orb seront assez abondantes pour qu'il ne soit nécessaire de faire appel aux eaux de l'Aude que dans le secteur dépendant de la Robine de Narbonne, où elles peuvent être épandues gravitairement et qu'en année d'hydraulicité déficitaire, l'utilisation par pompage des eaux sauvages de l'Aude dans les autres secteur's - utilisation par ailleurs très intéressante en ce qu'elle valorise les réserves de l'Orb et permet, par conséquent, une extension substantielle des surfaces irrigables - n'entraînera qu'une consommation d'énergie très modérée $(2.500 .000 \mathrm{kWh}$ environ). 


\section{RESEAU D'IRRIGATION DE LA ZONE OUEST}

Compte tenu des ressources ci-dessus analysées, le périmètre irrigable par le complexe OrbAude s'étendra - nous l'avons déjà dit - à 79.000 ha, en année sèche, type 1924. En année moyenne, cette surface pourrait être de beaucoup plus importante et déborder largement l'Hérault vers l'est. En fait, comme il ne saurait être question d'étendre au-delà de ce fleuve un système d'irrigation, dont le réseau devrait être conçu de telle façon qu'il puisse être alternativement irrigué par le complexe Orb-Aube et par le Rhône, le périmètre tributaire de ce complexe hydraulique sera limité à l'Hérault, ce qui conduit à une surface irrigable, en année moyenne, de 101000 ha.

De ces surfaces $(79.000$ ha en année sèche, type 1924, 101.000 ha en année d'hydraulicité moyenne), il y a lieu de déduire les 10.000 ha réservés à l'ouest de la zone d'action de la Compagnie, notamment dans le Minervois. Encore faut-il remarquer qu'une notable partie du Minervois, dont l'assèchement progressif entraîne une dégradation économique très alarmante, pourrait être assez facilement irriguée à partir du système de l'Orb, moyennant un relèvement par pompage à la cote 90 des eaux du canal principal de rive droite, qui, à leur arrivée à Ventenac, point où ce relèvement devrait avoir lieu, sont encore à la cote 50 .

Ce processus serait, sans doute, plus économique que celui jusqu'à maintenant envisagé par l'Administration pour l'irrigation du Minervois, et qui consiste à prélever les eaux de l'Aude à l'aval immédiat de Carcassonne pour les conduire dans la région d'Olonzac par un canal de $29 \mathrm{~km}$ de longueur.

Les surfaces irrigables ci-dessus indiquées ont été déterminées en fonction des modules d'irrigation ci-près :

\begin{tabular}{|c|c|c|c|c|c|c|}
\hline Mois & Avril & Mai & Juin & Juill. & Août & Sept. \\
\hline $\begin{array}{c}\text { Modules } \\
\text { en } \\
\text { l/s/ha }\end{array}$ & 0,0585 & 0,234 & 0,351 & 0,585 & 0,4095 & 0,117 \\
\hline
\end{tabular}

ce qui correspond à une dotation moyenne annuelle de $4.660 \mathrm{~m}^{3}$ par hectare. Il va de soi qu'il s'agit là de moyennes générales et que les modules d'irrigation devront être diversifiés par secteurs, en fonction des caractéristiques du sol, du mode d'irrigation et de la nature des cultures pratiquées. C'est ainsi, par exemple, que les basses plaines dépendant de la Robine de Narbonne, sur lesquelles se pratique la culture du riz, devront recevoir des dotations très supérieures à celles moyennes ci-dessus indiquées.

Les eaux seront dérivées à Lugné par un barrage de prise dont le seuil fixe déversant sera arasé à la cote 65,00, qui correspond à celle minimum nécessaire pour l'alimentation du canal principal. Ce barrage relèvera, par conséquent, de $14 \mathrm{~m}$ le plan d'eau d'étiage. Il sera, par la suite, équipé de 6 vannes-segments de $13 \mathrm{~m}$ $\times 3 \mathrm{~m}$ à commande électrique et de 2 vannesclapets à commande hydraulique automatique. Cette bouchure mobile permettra de remonter le plan d'eau à la cote 68,00 , tout en assurant l'évacuation des crues - dont le débit maximum est susceptible d'atteindre $2.500 \mathrm{~m}^{3} / \mathrm{s}$ — sans surélévation excessive de la ligne d'eau à l'amont.

La tranche utile de la retenue, comprise entre les cotes 65,00 et 68,00 et dont le volume est de 1 million de mètres cubes, servira de réserve compensatrice. Elle permettra, dans une première phase de l'aménagement, de laminer les lâchures de la centrale de Montahut exploitée en usine de pointe de façon à assurer une plus complète alimentation de la station de pompage destinée à refouler les eaux de l'Orb dans le réservoir de Rieuberlou, en attendant que le remplissage de celui-ci puisse être assuré par gravité à partir du barrage d'accumulation de Ceps. Elle donnera, par la suite, une plus grande souplesse de fonctionnement à la centrale de Lugné, dans laquelle seront turbinées les eaux de l'Orrb, dérivées à Ceps et transitant par le Rieuberlou, l'usine pouvant ainsi n'être pas astreinte à travailler au débit constant exigé par l'irrigation. Les deux vannes-clapets automatiques de $13 \times 6 \mathrm{~m}$ obtureront deux pertuis, dont le seuil fixe sera arasé à la cote 61,00 , soit à $2 \mathrm{~m}$ audessous des seuils de prise, qui seront ainsi maintenus libres de tout engravement. I'un de ces pertuis de chasse sera installé en rive gauche pour le dégagement de la prise du canal principal d'irrigation. L'autre le sera en rive droite, à l'aval de l'ouvrage par lequel, en première phase, les eaux de l'Orb seront amenées à la station de pompage destinée au remplissage du Rieuberlou et par lequel, en deuxième phase, les eaux dérivées à Ceps et turbinées en pied du barrage de Rieuberlou seront rendues à la rivière, à l'amont du barrage de prise, pour ètre finalement entonnées dans le canal principal d'irrigation.

Ce canal, dont le débit maximum sera de 
$38,5 \mathrm{~m}^{3} / \mathrm{s}$, se développera d'abord sur $7.700 \mathrm{~m}$, en rive gauche de l'Orb, qu'il franchira par une bâche de $236 \mathrm{~m}$ de longueur à l'entrée des gorges de Réals. A la sortie des gorges, le canal desservira la centrale de Réals d'octobre à avril, c'est-à-dire en dehors de la période des irrigations et, si les débits disponibles le permettent, durant les premiers mois de cette même période, alor's que la satisfaction des besoins agricoles n'absorbe pas intégralement la capacité de transport de l'adducteur.

Après le piquage de l'usine, une partie du débit ( $12 \mathrm{~m}^{3}$ en pointe) sera renvoyée par siphon en rive gauche, en vue d'alimenter la branche maîtresse commandant l'irrigation de toute la partie du périmètre située entre Orb et Hérault. Le débit complémentaire $(26,0 \mathrm{~m} / \mathrm{s})$ sera réservé à l'irrigation des plaines situées en rive droite. Le canal correspondant, après s'être dégagé de la vallée de l'Orb par un tracé comportant quelques souterrains, jusqu'à Cazouls-les-Béziers, se développera entre les cotes 60 et 50 , en direction de l'ouest, parallèlement au canal du Midi qu'il rejoint à Capestang et à une vingtaine de mètres au-dessus de lui. Il domine ainsi pratiquement toutes les terres intéressantes comprises entre le cours de l'Aude et les collines qui limitent par le sud la vallée du Lirou. Un certain nombre de secondaires s'en détachent, dont le plus important, ayant son origine près de Quarante, se prolongera jusqu'à l'Aude par le canal d'atterrissement de Capestang et au sud de ce fleuve (franchi à niveau à l'amont du barrage de Moussoulens), par la Robine de Narbonne, jusqu'aux étangs de Bages et de l'Ayrolle.

A son arrivée, à la cole 50 , à Ventenac, le canal principal est encore susceptible de desservir, par gravité, les belles plaines : Villedaigne, Ornaisons, Cruscade Luc-sur-Aude et Lézignan, qui s'étendent, sur 8.000 ha environ, en rive droite de l'Aude. L'irrigation de ces terres exigera, néanmoins, la traversée de ce fleuve entre Ventenac et Laparre, par un siphon de $5.300 \mathrm{~m}$ de longueur, composé de deux tubes de $1,60 \mathrm{~m}$ de diamètre.

La surface totale desservie en rive droite de l'Orb sera ainsi de 51.000 ha. Elle sera irriguée enticrement par gravité, hormis 2.300 ha environ, dans la région de Puisserguier, qui ne pourront être atteints qu'au prix d'un pompage, d'ailleurs modéré ( $35 \mathrm{~m}$ environ).

\section{TRAVAUX D'ASSAINISSEMENT ET DE PROTECTION CONTRE LES INONDATIONS}

Nous avons déjà signalé, à l'occasion de l'examen rapide que nous avons fait des ressources en eau susceptibles d'être tirées des fleuves côtiers, que sur la Vidourle, l'Hérault et l'Orb, des harrages étaient envisagés, dont les retenues devaient permettre de protéger les basses vallées correspondantes contre les inondations.

Parallèlement à ces ćquipements et à la reforestation nécessaire mais aux effets nécessairement lents, de la partie supérieure des bassins versants, il conviendra de faciliter l'écoulement à la mer des eaux, qui actuellement stagnent dans certaines zones littorales. Le couloir de Saint-Gilles, compris à l'est de cette ville, entre le pied des Costières du Gard et le Petit-Rhòne, a déjà fait l'objet, de la part de l'Administration du Génie rural, d'importants travaux d'assainissement, qui seront complétés par la Compagnie à l'occasion de la construction du canal principal de Fourques à Pichegu.

Mais il entre, en outre, dans les intentions de la Compagnie de dresser le projet des travaux indispensables pour assainir la Petite Camargue et les terres basses situées de part et d'autre du cours inférieur du Vidourle, et de rechercher les concours financiers nécessaires à leur réalisation. L'absence ou l'insuffisance du drainage de ces terres fail qu'elles sont incultes ou mal utilisées. En bordure de l'étang de Maugio, la salure des sols augmente et s'étend vers le nord. Il en est de même en maints autres points du littoral et notamment dans les terres qui bordent le cours inférieur de l'Orb. Il est évident que dans ces secteurs le drainage devra précéder l'irrigalion, même quand la principale culture envisagée - c'est le cas de la Petite Camargue - est le riz, qui s'accommode, dans une certaine mesure, d'une faible salinité du sol. Un projet a déjà été dressé qui, si les concours financiers nécessaires à sa réalisation sont trouvés, améliorera très sensiblement les conditions actuelles d'écoulement du Vidourle à la mer. Un plan d'assainissement de la Petite Camargue est, d'autre part, en voie d'élaboration. L'absence quasi totale de relief interdit de penser qu'un drainage efficace de cette région puisse être assuré par gravité. Les eaux devront être rejetées au PetitRhône, par pompage, à Sylveréal, où il est normal de faire converger les principaux axes de drainage des terres marécageuses situées au sud du canal de navigation du Phône à Sète, entre Franqueveau et Aigues-Mortes.

Dans cette région, où les maxima pluviométriques journaliers observés atteignent couram- 
ment $78 \mathrm{~mm}$ et peuvent s'élever à $170 \mathrm{~mm}$, les débits à pomper seront assez élevés, nonobstant l'écrêtement résultant de la submersion temporaire tolérable par les cultures. Il est probable que l'on sera conduit à compter sur un apport unitaire maximum de $3 \mathrm{l} / \mathrm{ha} / \mathrm{s}$. A tout le moins, les axes de drainage et le Génie civil des stations de pompage devront être prévus pour ce débit, les pompes pouvant n'être installées que progressivement, au vu des premiers résultats enre- gistrés et au lur et à mesure du développement du réseau de drainage.

Il est probable, d'autre part, que l'on sera amené à envisager la récupération pour l'agriculture d'une partie des espaces actuellement à l'état de lagunes, les plans d'eau résiduaires étanl aménagés à bords francs et équipés, suivant l'exemple donné en Vénétie par les Italiens, en vue d'une organisation rationnelle de la pèche.

\section{SITUATION ACTUELLE DES TRAVAUX}

Le canal principal issu du Rhône a été attaqué en janvier 1957, sur un tronçon de $7 \mathrm{~km}$ compris entre la station de pompage de Pichegu et Saint-Gilles. Les terrassements correspondants, qui comportaient l'exécution de $1.200 .000 \mathrm{~m}^{3}$ de déblais, sont à peu près terminés et vont se poursuivre sur un lot nouveau, de $6 \mathrm{~km}$, prolongeant le premier vers l'aval. Les revêtements et les ouvrages d'art, au nombre desquels figure un pont-canal en béton précontraint de $200 \mathrm{~m}$ de portée totale, en huit travées de $25 \mathrm{~m}$ solidaires 4 par 4 , sont en cours d'exécution.

Les travaux relatifs au troncon de $12 \mathrm{~km}$, allant de la prise au Rhône à Pichegu, ont été adjugés fin juillet. Les gros engins de terrassement nécessaires à l'exécution de ce lot ont été rapidement approvisionnés et montés, et l'ouverture du chantier est imminente.

Le matériel électromécanique correspondant à la première phase d'équipement de la station de pompage de Pichegu a été commandé, et les premiers groupes de pompage doivent pouvoir etre mis en service à la fin du printemps de 1959.

Un premier lot de la branche maîtresse du réseau des Costières et de la Vistrenque a été attaqué le mois dernier.

Le génie civil de la slation de pompage de
Pichegu et l'ouvrage de prise au Rhône ont été mis au concours. La dévolution des travaux correspondants interviendra courant novembre.

La construction d'un lot de $13 \mathrm{~km} \mathrm{du}$ canal principal, compris entre Saint-Gilles et le Vidourle, sera mis en adjudication avant la fin du mois.

Dans le secteur ouest, on peut espérer qu'avant la fin de l'année en cours, des marchés auront pu être conclus pour la construction d'un premier lot du canal principal issu de l'Orb, de l'ouvrage de prise de Lugné el peut-être aussi d'un des barrages d'accumulation nécessaires.

Dès le milieu de l'année prochaine, par conséquent, l'ossature maîtresse du réseau d'irrigation commencera à bien se dessiner sur le terrain, Dans l'intervalle, aura été adjugé l'équipement, en Costières, d'un premier lot du réseau de distribution. Il a été convenu que ce réseau serait en charge, en vue de permettre l'irrigation à la demande et l'alimentation directe de sprinklers à basse pression. Il a été admis, en effet, que tout au moins en Costières, le relief du sol et plus encore la nature assez perméable de celuici, justifiaient le recours au mode dirrigation par aspersion, relativement coûteux de premier établissement, mais qui ménage l'eau et dont l'emploi, aux U.S.A. et en Italie notamment, tend à se généraliser.

\section{CONCLUSION}

On peut juger, par ce qui précède, de l'ampleur de la tâche dévolue à la Compagnie Nationale d'Aménagement de la Région du Bas-Rhône et du Languedoc.

Pour la mener à bien il faudra, non seulement des moyens financiers importants et une grande continuité de vues, que la création d'une Com- pagnie spécialisée a, entre autres raisons d'être, celle d'assủrer, mais aussi la collaboration confiante de tous les intéressés et principalement de ceux-là même qui seront finalement appelés à bénéficier de la transformation économique projetée.

Si la Compagnie a comme but essentiel d'ir- 
riguer et de permettre ainsi au paysan languedocien de sortir de l'impasse où il se trouve actuellement engagé, du fait du caractère désormais non rentable d'une culture qu'il ne peut, faute d'eau, remplacer par une autre, elle poursuit également d'autres objectifs s'inscrivant dans le cadre plus large de la rénovation économique du Bas-Rhône-Languedoc.

Et pour ces tâches économiques, dont elle n'a d'ailleur's pas l'exclusivité, elle entend n'intervenir directement que le moins possible, estimant plus efficace d'analyser les données du problème, de tracer des lignes d'action, de susciter les concours techniques et financiers nécessaires, en un mot de galvaniser toutes les bonnes volontés, que de réaliser elle-même, dans une atmosphère sans écho, quelques équipements fragmentaires dont l'ampleur ne pourrait être que limitèe.

Le succès de l'œuvre dépendra de la collaboration confiante et permanente entre les administrations, les collectivités publiques, les corps élus, l'initiative privée et la Compagnie. Il dépendra aussi de la répartition harmonieuse des di- verses tâches à accomplir entre les organismes tendant à la réalisation d'objectifs communs.

L'œuvre entreprise est certes importante, mais elle a des précédents, et s'inscrit parfaitement dans la ligne aujourd'hui bien tracée des aménagements régionaux. Flle reste modeste au regard d'autres cuvres de même nature entreprises à l'étranger et dont l'équipement du sud de I'Italie par la Cassa del Mezzogiorne est un exemple vivant. Il serait paradoxal que la France ne fasse pas en Languedoc l'effort d'équipement nécessaire pour assurer à cette province un écquilibre économique satisfaisant, alor's que les pays méditerranéens voisins n'ont pas hésité à engager des travaux d'irrigation considérables pour permettre à certaines de leurs provinces de sortir du marasme auquel des conditions naturelles défavorables, l'aridité du climat en particulier, avaient jusqu'à maintenant condamné l'agriculture.

En s'engageant sur une voie nouvelle et constructive, notre pays apportera à la jeunesse languedocienne, avec un niveau de vie plus élevé, la confiance en l'avenir.
M. le Président remercie M. Bayzr de son intéressanto communication, et remarque qu'elle est tont a fait dans le style souhaitable pour la S.H.F., qui s'efforce d'intéresser ses membres aux grands travaux avant que ceuxci soient entrepris. D'autre part, le site de Ceps est très remarquable.

Sur la demande de M. Flancroos (Société Seuralite), M. Bauzil rappelle quelques-unes des caractéristiques des barrages prévus dans le projet et donne des rensei- gnements complémentares sur l'ordre de leur exécution seul le barrage de dérivation et de compensation de Lugné, sur l'Orb, va etre commencé en 1958, et on espère engager un barrage d'accumulation des l'année 1959; le barrage de Rieuberlou comporte un barrage principal dont la retenue est à la cote 130 , et un ouvrage annexe destiné à barrer un col qui se trouve en amont à la cote 115 .

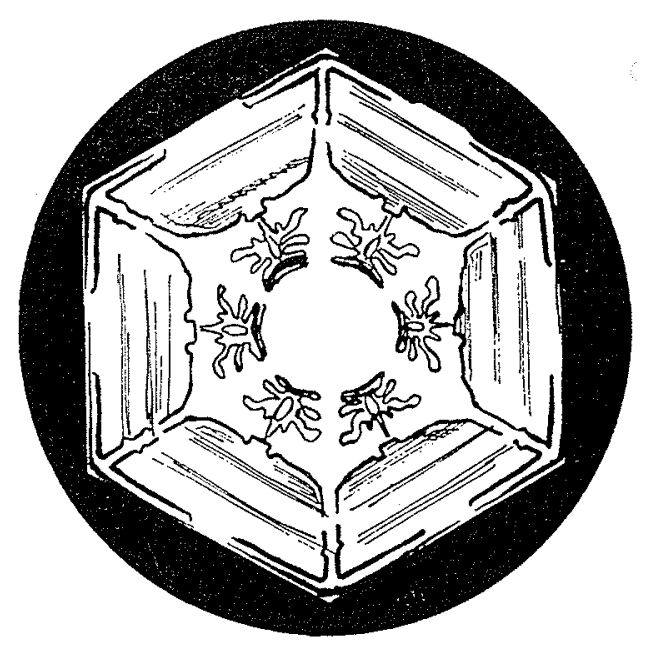

